\title{
COMPOSITION OF SOLAR COSMIC RAYS
}

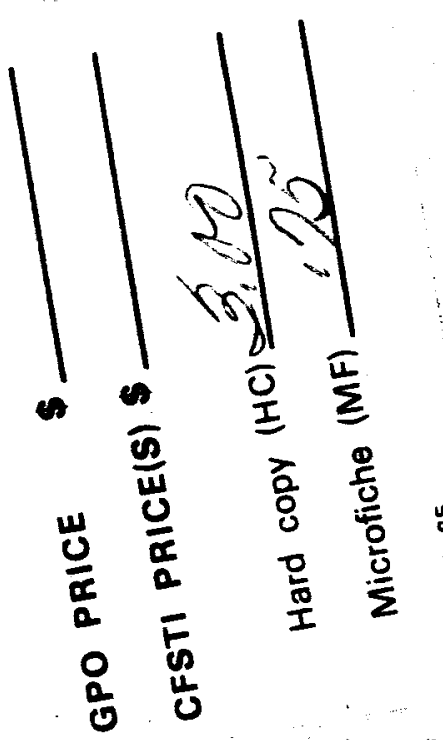

\section{MAY 1965}

\section{NA SA}

\section{S. BISWAS}

C. E. FICHTEL
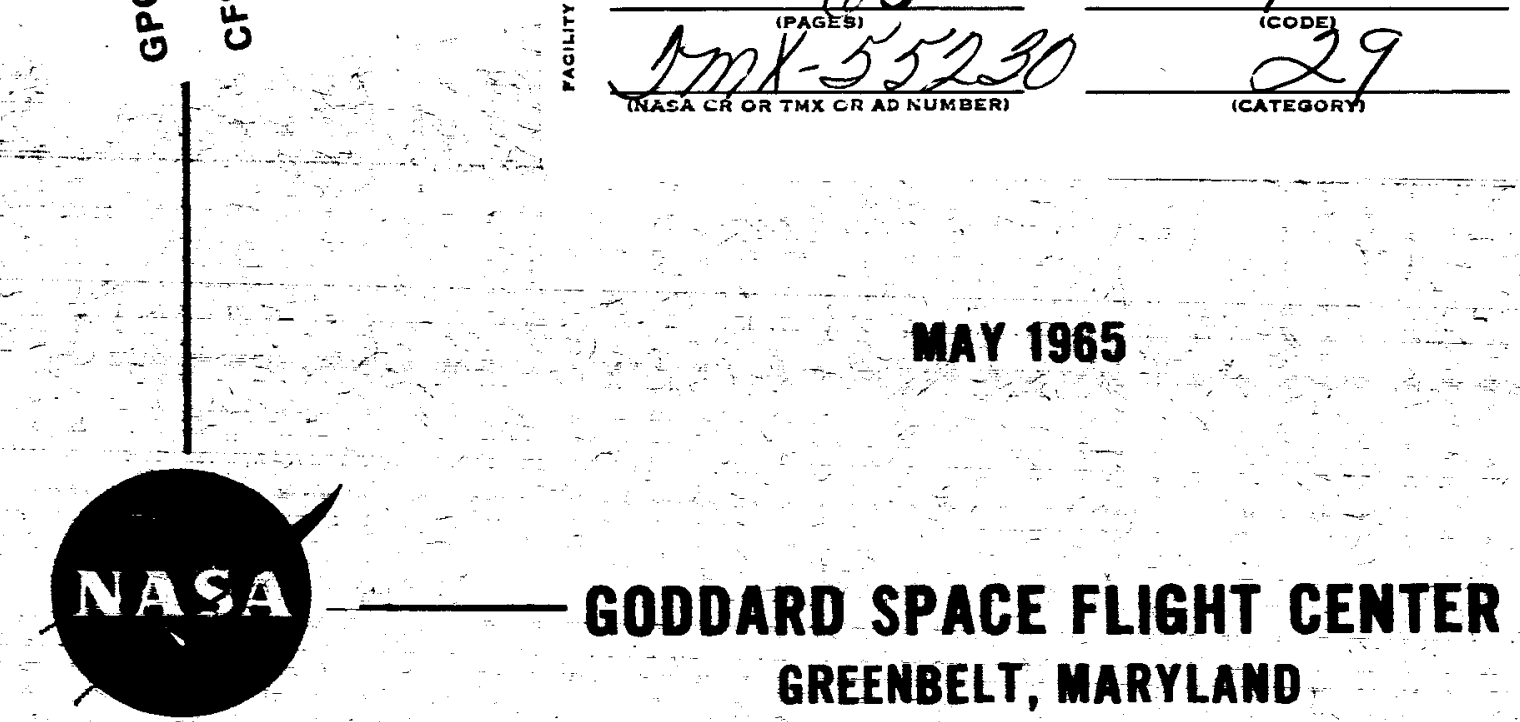
GREEMBELT, MARYLAMD

Submitted to Spoce Science Reviews Energetic Particles Preprint Series 


\author{
COMPOSITION OF SOLAR COSMIC RAYS \\ S. Biswas \\ Tata Institute of Fundamental Research \\ Bombay, India \\ C. E. Fichte1 \\ NASA/Goddard Space Flight Center \\ Greenbelt, Maryiand
}

\title{
I. INTRODUCTION
}

For over two decades, it has been known that the sun is capable of accelerating particles to high energies in association with major solar disturbances. The existence of these energetic particle events was first realized by Forbush (1946) as a result of ground level observations; subsequentiy, the results of several experiments have shown that there are many of these events and that they occur most frequently during the years of high solar activity (e.g. McDonald, 1963; Roederer, 1964; and Webber, 1964). The known events have varied in size from those whose intensities are just barely detectable to events where, for one day, the flux of particles with energies greater than $20 \mathrm{Mev}$ exceeded $10^{3}$ particles/( $\mathrm{cm}^{2}$ ster.sec.). The normal non-solar cosmic ray intensity above $20 \mathrm{Mev}$ is about 0.2 particles $/\left(\mathrm{cm}^{2}\right.$ ster.sec.). Although protons are the most numerous particles in a given velocity interval, many other types of particles are known to exist, and their presence, energy spectra, and relative abundances are of considerable importance not only in the study of solar cosmic rays, but also solar physics and non-solar cosmic rays.

The study of the composition of solar cosmic rays was pursued with considerabie success during the wost recent solar maximum (approximately 
. 1957 to 1962). Already many of the general features seem to be clear, and some detailed knowledge is emerging. Detailed studies of the proton energy spectra as a function of time now exist for several events from 3 to $600 \mathrm{Mev}$ as a result of the scintillation counter experiments flown on Explorers XII and XIV (Bryant, Cline, Desai and McDonald, 1962; 1963). In addition less complete time histories of solar proton spectra exist for some earlier events from measurements made with simple electronic detectors and nuclear emulsions flown on balloons, sounding rockets, and sateliites (e.g. McDonald, 1963.). The energy spectra of solar protons and helium nuclei have been measured simultaneously on several occasions using nuclear emulsion detectors flown on balloons (Biswas, Freier and Stein, 19є2; Biswas and Freier, 1961; Ney and Stein, 1962; Freier and Webber, 1963; Freier, 1962; Freier, 1963) and in rockets (Biswas, Fichtel and Guss, 1962; Biswas, Fichtel, Guss and Waddington, 1963). In spite of their limited number, these measurements are of particular interest because the different charge to mass ratio of $\mathrm{He}^{4}$ and $\mathrm{H}^{1}$ permits the separation of velocity and rigidity (momentum/ charge) effects. Turning to the isotopic composition, detectable fluxes of deuterons have been seen in two events (McDonald, Balasubrahmanyan, Brunstein, Hagge, Ludwig, and Palmeira, 1965) and relatively severe upper limits have been set in two others (Biswas, et al., 1963 and Freier and Waddington, 1965). Results on the presence of tritium and $\mathrm{He}^{3}$ in the solar particle radiation have been obtained by Fireman, DeFelice, and Tilles (1961), Tilles, DeFelice and Fireman (1963) and Schaeffer and Zahringer (1962). 
Smaller quantities of nuclei with higher charges have also been seen in several events (Fichtel and Guss, 1961; Biswas, Fichtel, and Guss, 1962 ; Yagoda, 1961; Pomerantz and Witten, 1962 ; Kurnosova, Razorenov, and Fradkin, 1962; Biswas et a1., 1963; Biswas and Fichtel, 1962; and Biswas et al, 1965), and it seems likely that these heavier nuclei are always present since they have been observed every time the intensity of the event was sufficiently great to expect to be able to detect them on the basis of their abundances in other events. Detailed charge studies (Biswas, et al., 1963; Biswas and Fichte1, 1963) seem to indicate that the multiply charged component (the nuclear charge $z>2$ ) has a composition similar to that of the sun, and hence dissimilar to that of the cosmic radiation coming from beyond the solar system. Energetic electrons are very rare (Ear1, 1962; Yates, 1963; Ney and Stein, 1962; Cline, Ludwig, and McDonald, 1964), and positive evidence for them exists in only one solar cosmic ray event (Meyer and Vogt, 1962), and even then their intensity was very low compared to that of the protons. There is no direct evidence for neutrons.

This review of solar particle composition will begin with a summary of our present knowledge of the charge spectrum of the nuclear component, the energy spectra of the various nuclear components, the isotopic composition of the hydrogen and helium nuclei, and the available information on other possible components such as electrons and neutrons. This will be followed by a discussion of the relation of these measurements to the theories of solar particle propagation and acceleration. 
Finally, the insight which these results can give into the relative abundances of elements on the sun and the origin of the cosmic rays coming from beyond the solar system will be discussed

II. EXPERIMENTAL OBSERVATIONS ON THE COMPOSITION OF SOLAR COSMIC RAYS

This section will be divided thus: First, the charge spectrum of the solar nuclei and the energy spectra of different nuclear charge groups will be discussed. Then, the available data on the isotopic composition of the two principal charge species, hydrogen and helium, will be presented. Finally, the experiments to detect other components will be summarized.

A. CHARGE SPECTRUM OF SOLAR NUCLEI AND THEIR ENERGY AND RIGIDITY SPECTRA

At the present time it seems to be meaningful to speak of the charge composition of multiply charged nuclei, but there seems to be no simple relationship between the abundances of protons and the nuclei of higher charge. This picture develops from the similar composition, independent of energy, of the multiply charged nuclei at different times and in different events, and the correspondingly large variations in the proton to helium ratio. The remainder of this section, IIA, will be devoted principally to the analysis of the complicated proton-helium problem and a description of the energy and rigidity spectra. A detailed discussion of the charge spectrum of the multiply charged nuclei will be left until the next section, IIB.

The data for the study of the solar nuclei, much of which is summarized in Tables 1 and 2 , comes from a variety of detectors and 
experiments flown on balloons, sounding rockets, and satellites. In the case of balloon borne detectors, the lowest energy particle which can be measured is determined either by the air cutoff (typically 75 Mev for protons) or in some cases by the geomagnetic cutoff. In general, the lower limit of the detectable energy in sounding rocket experiments flown from regions close to the magnetic poles and satellite experiments is caused by the detector system itself and is therefore usually $10 \mathrm{Mev}$ or less.

Since the particle distribution that is being examined consists not only of nuclei of different types, but also nuclei of different velocities, it is necessary to examine both the composition and the velocity distribution as a function of time in an event. In addition, possible event to event variations must also be considered before meaningful statements about the relative abundances can be made. Finally, it is desirable to proceed even further and separate the effects of velocity and rigidity because in general the acceleration and the propagation of solar cosmic rays could depend on both of these parameters.

In proceeding to analyze the data, it must be remembered that the charge to mass ratio of the proton differs by a factor of two from most of the nuclei of higher charge, such as $\mathrm{He}^{4}, \mathrm{C}^{12}, \mathrm{O}^{16}$, and $\mathrm{Ne}^{20}$, and, therefore, for a given velocity, the rigidity of a proton is less than the other nuclei. Here, we are assuming that the helium nuclei observed are mostly $\mathrm{He}^{4}$. 
- For those times when data on both hydrogen and other nuclei exist it is possible to find simple mathematical expressions for both the energy per nucleon and the rigidity spectra in the limited intervals where data exist for both types of particles being considered.

These representations are as follows:

$$
\begin{aligned}
& d J / d W=c(A, Z, t)\left(W / W_{0}\right)^{-Y(A, X, t)}, \\
& d J / d R=K(A, Z, t) \exp \left[-R / R_{0}(A, X, t)\right],
\end{aligned}
$$

where $J$ is the integral particle flux, A the atomic number, $Z$ the nuclear charge, $t$ the time in the event, $W$ the total energy, $W_{0}$ the rest energy, and $R$ the rigidity. Although these equations are adequate representations for the limited intervals where nuclear composition data exists, it should be mentioned that neither equation (1) nor (2) holds for all energies and times, although equation (2) is better than equation (1) for energies below about $35 \mathrm{Mev}$. Measured values of $v$ and $R_{0}$ are given in tables 1 and 2 for those instances where data are available for more than one component.

Freier and Webber (1963) were the first to show that an exponential rigidity spectrum of the form given by equation (2) was an excellent representation of the data. They showed further that the rigidity spectra of protons and helium nuclei are often similar in shape; that is $R_{0}$ of equation (2) is approximately the same for the two nuclear species. An example of this effect is shown in Fig. 1. However, they are not always exactly the same. In two instances, at 1603 UT on November 19,1960 and at 1405 UT and 0930-1700 UT on September 3, 1960, when the spectra of protons and multiply charged particles could be measured over a wide rigidity 
interval, the $R_{0}$ values were significantly different. An analysis of the data (Biswas and Fichtel, 1963) indicated that there was less than a $0.1 \%$ chance of $R_{0}$ values of protons and helium nuclei being the same. Fig. 2 is an example of spectra which have different $R_{0}$ values. The measurements were made using nuclear emulsions flown on balloons and sounding rockets and the references are given in table 2 . In the balloon experiments, the measurements covered an energy range from about 300 to $800 \mathrm{Mev}$ for the protons and about 80 to $300 \mathrm{Mev}$ inucleon for the helium nuclei, while in the latter studies protons and helium nuclei were measured in the energy intervals from about 15 tp $300 \mathrm{Mev}$ and 30 to $130 \mathrm{Mev}$ inucleon respectively.

When proton to helium ratios are compared, it is found that, although the helium and hydrogen nuclei seem to have similar rigidity spectra, the ratio of the two components in the same rigidity interval varies greatly from one event to another and from one time in an event to another. Above approximately $0.8 \mathrm{Mev}$ rigidity, the proton to helium ratio has varied from $\geq 50: 1$ to $1: 1$ with smaller ratios tending to be more common. Table 2 gives some of the ratios that have been observed by experimenters. These data are taken largely from the summaries of Freier and Webber (1963) for the rigidity range above approximately $0.8 \mathrm{Bv}$ and $\mathrm{Biswas}$ and Fichtel (1963) for the rigidity range from approximately 0.5 to $1.0 \mathrm{Bv}$. Additional measurements included in Table 2 were made recently by McDonald, et al. (1965) using a scintiliator telescope flown on the IMP satellites.

In all the instances where the fluxes of the proton and helium nuclei could be measured in the same energy per nucleon interval, the 
He nuclei energy/nucleon spectrum had a much steeper slope than the proton one. Fig. 3 shows a typical example of this effect and Table 1 gives the $V$ values calculated from Equation (1). Figures $4 \mathrm{a}$ and $4 \mathrm{~b}$ show the energy/nucleon spectra for protons, helium nuclei, and medium nuclei for those instances when all three nuclear species have been observed at the same time.

In considering the hydrogen to helium nuclei ratio within a given velocity increment, then, the interval must be specified since the ratio varies markedly with energy, as shown in Fig. 5. In addition to this ratio varying appreciably with energy per nucleon in one event, there is now some evidence to indicate that for a given velocity interval there can also be considerable variation in this ratio from one event to another as shown in Fig. 5. Table 2 gives the ratios which have been measured at times when comparisons could be made in the same energy per nucleon interval. Unfortunately, the high and uncertain magnetic cutoff at Minnesota prevents the calculation of this ratio in many cases when helium nuclei data were available because the protons in the same kinetic energy interval as the observed He nuclei were partially or entirely forbidden by the magnetic field.

\section{B. MULTIPLY CHARGED NUCLEI}

The data on heavy nuclei $(z \geq 3)$ are more limited but easier to summarize. Because of their short ranges, almost all of the information on energetic solar heavy nuclei has come from sounding rockets and satellites, and in only two cases has it even been possible to detect them at balioon altitudes. Heavy nuclei were first detected in the 
September 3, 1960 solar cosmic ray event in nuclear emulsions flown on sounding rockets from Fort Churchill (Fichtel and Guss, 1961). They were subsequently seen again in the November 1960 events in nuclear emulsions flown on balloons, sounding rockets, and satellites by Biswas et al. (1962), Yagoda et al. (1961), Ney and Stein (1962), Biswas et al. (1963), and Biswas and Fichtel (1963) with an ionization and Cerenkov counter system flown by Pomeranz and Witten (1962), and in the July 18, 1961 event with nuclear emulsions flown on a balloon (Biswas, et al., 1965).

The evidence which exists at present indicates that the energy per nucleon spectra of the medium nuclei and the helium nuclei are the same. The $Y$ values of Equation 1 for helium and medium nuciei as calculated by Biswas and Fichtel (1963) are given in Table 1 and are seen to be the same within uncertainties. Further, since all of the medium nuclei which occur in detectable amounts have the same charge to mass ratio as $\mathrm{He}^{4}$, the rigidity spectra of these two nuclear species are also the same, assuming of course that the nuclei have been completely stripped of their electrons. Figs. 1, 2, 3, and 4 all show the similarity of the helium and medium spectra. In addition to having the same rigidity spectra, the relative abundances of helium and medium nuclei in the same rigidity intervals have been measured several times in three events and found to be the same within uncertainties, as indicated in Table 3. The variation of one of the helium to medium ratios from the average by two standard deviations is not thought to be significant both because one such deviation in a sampie of six is not 
too unlikely and because the other two ratios measured in the same event are within one standard deviation. Since the helium to medium ratio is constant, the proton to medium ratio varies in a manner similar to the proton to helium one, which was shown in Fig. 5.

The relative abundances among the heavy nuclei for those nuclei which could be measured in the same energy per nucleon intervals have been found to be the same each time a measurement was made, namely, five times in two events (Biswas et al., 1962 and Biswas et al., 1963). These data are summarized in Table 4 . This result, of course, suggests that if these other nuclei had been present in sufficient numbers to measure a rigidity spectrum, their rigidity spectrum would have been the same as the medium and helium nuclei.

The results summarized in the last two paragraphs indicate that it is possibly meaningful to speak of relative abundances of solar cosmic ray multiply charged nuclei, and that the best estimates of these abundances would be obtained by taking the average composition in the same velocity intervals from all of the data available. Therefore, the average composition of the multiply charge nuclei in the same velocity intervals as measured in the five flights mentioned above is presented in Table 5 with a base of one having been chosen for oxygen. Among the heavy nuclei (those with nuclear charges greater than two), the medium nuclei ( $6 \leq$ nuclear charge $\leq 9)$ are seen to be the most abundant, while $B e$ and $B$ are so rare that only upper limits can be set. A closer examination shows that the relative abundances of the energetic solar particles are the same within uncertainties as the solar abundances determined by spectroscopic means. Since the 
solar and universal abundances are similar, although not the same, the solar cosmic ray composition is also similar to the universal abundances. The solar cosmic rays are, however, markedly different in composition from the galactic cosmic rays which are weil known to be rich in the heavy elements. The possible significance of these results will be discussed in some detail in the next two sections of this paper, i.e. III and IV.

Kurnosova, Razorenov, and Fradkin (1962) have reported a few increases of heavy nuclei above cosmic ray background in the relativistic region lasting on the order of two to twenty minutes and not associated with a solar particle event of the usual variety. These measurements were made on a satellite with a Cerenkov counter system. Although other experiments which would have detected this type of increase have been flown (McDonald, 1965), no other increase of relativistic heavy nuclei hàs been reported. Since these events are apparently of a different nature from the low energy solar particle events being discussed here and nothing more is yet known about them, they will not be treated further.

c. ISOTOPIC COMPOSITION OF SOLAR HYDROGEN AND HELIUM NUCLEI As mentioned earlier, protons are by far the most abundant singly charged nuclei. However, experiments have been performed to look for the possible presence of deuterons and tritons. Attempts to detect deuterons have been made in four events. In a rocket flight on November 12,1960 , Biswas et al. (1962) found the deuteron to proton ratio was less than $3 \times 10^{-3}$ in the energy interval from 25 to $48 \mathrm{Mev}$, nucleon and subsequently Freier and Waddington(1964b) found a 1imit of 
$2 \times 10^{-3}$ in the interval above $50 \mathrm{Mev} /$ nucleon in the July 18,1961 event. Both of these measurements were made with nuclear emulsions. McDonald et al. (1965) found the proton to deuteron ratio to be about $5 \times 10^{-3}$ for protons with energies from 15 to $75 \mathrm{Mev}$ and deuterons with energies from 20 to $100 \mathrm{Mev}$ in both the March 16, 1964 and February 5, 1964 events. Since the particle energy spectra decreases rapidly with energy, the proton to deuteron ratio in the same energy. nucleon interval is somewhat less than this number, but still finite These particles were detected in the IMP scintillation counter telescope mentioned previously. Perhaps it is important to note that the latter two events in which deuterons were actually seen were small events during a period near solar minimum, whereas the other two events were much larger ones.

Small quantities of tritium were detected by radiochemical studies of materials recovered from a satellite and sounding rockets flown in the November 12, 1960 event (Fireman et al., 1961 and Tilles et al., 1963). The observed particles represent the combination of tritons formed in interactions in the material above the detector, tritons temporarily trapped in the radiation beits, and any primary tritons which might be present. In trying to make a quantitative estimate of the triton abundance, the situation is further complicated by having no direct measure of the integrated proton flux in the case of the satellite, Discoverer 17. Assuming the majority of the tritons are 
solar, as Tilles et al. suggest, and an integrated proton flux above $30 \mathrm{Mev}$ of between $10^{9} / \mathrm{cm}^{2}$ (estimated by the authors on the basis of riometer data and direct particle measurements at a few times during the event) and $4 \times 10^{9}$ (estimated by Lal, Rajagopalan, and Venhatavaradan, 1963, from radiochemical data) a triton to proton ratio of (1 to 4) $x$ $10^{-3}$ above $30 \mathrm{Mev} /$ nucleon is obtained. Lal et al. (1963), on the basis of radioactivity data, concluded that more than $80 \%$ of the tritons were secondaries, in which case the ratio might be as small as $2 \times 10^{-4}$. In a sounding rocket payload Tilles et al. (1963) measured an integrated triton flux of about $8 \times 10^{4} / \mathrm{cm}^{2}$ in the energy interval from approximately 0 to $27 \mathrm{Mev} /$ nucleon. From the scintillation counter results of Ogilvie et al. (1962), the integrated flux of protons in the energy interval from 2 to $27 \mathrm{Mev}$ was about $8 \times 10^{7} / \mathrm{cm}^{2}$ during the rocket flight. Therefore, the ratio $\mathrm{H}^{3} / \mathrm{H}^{1}$ is $\leq 10^{-3}$ for solar particles in the energy range from approximately 0 to $27 \mathrm{Mev} /$ nucleon. Direct measurements by Biswas et al. (1962) in nuclear emulsions flown in the same sounding rocket on November 12 , set an upper limit to the triton flux in the interval from 20 to $45 \mathrm{Mev} /$ nucleon of $<.7 \mathrm{H}^{3} / \mathrm{cm}^{2}$ ster sec. The flux of protons measured in the same flight (Biswas et al., 1962) was $0.9 \times 10^{3}$ protons $i \mathrm{~cm}^{2}$ ster sec in the same interval (20-45 Mev). Therefore, the triton to proton ratio was $\leq 10^{-3}$ in the energy interval 20-45 Mev/nucleon. In the July 18, 1961 event, Freier and Waddington (1964b) using nuclear emulsions flown on a balloon, set an upper limit to the triton to proton ratio of $0.6 \times 10^{-3}$ in the energy region above $50 \mathrm{Mev} /$ nucleon. 
On the basis of the above analysis, we feel that the present experimental results indicate that the deuteron to proton ratio for solar particles in the approximate interval from 10 to $10^{2} \mathrm{Mev} /$ nucleon is on the order of a (few) $\times 10^{-3}$ or less and the triton to proton ratio is probably less than $10^{-3}$. Variations in these ratios from event to event probably occur; so there is nothing too surprising about the limit in one event being more severe than the ratio measured in another. The ratio might also be expected to vary with the velocity interval.

Relatively little is known for certain about the relative abundance of the helium isotopes. Schaeffer and Zahringer (1962) reported the presence of $\mathrm{He}^{3}$ in a radiochemical study of some of the components of the Discoverer 17 satellite and estimated the $\mathrm{He}^{3} / \mathrm{He}^{4}$ ratio to be 0.2 with an uncertainty of a factor of three. However, they observed larger concentrations of $\mathrm{He}^{3}$ at greater depths of material. This result is surprising if the majority of these particles are primaries because the steep energy spectrum should have predominated over any effect of the variation of the geomagnetic cutoffe with satellite position to give a decreasing $\mathrm{He}^{3}$ flux with depth. Further, the abundance of $\mathrm{He}^{3}$ in the experiment mentioned corresponds to a flux which seems to be about ten times larger than the total helium flux in the energy range from 40 to $105 \mathrm{Mev} /$ nucleon estimated from the work of Biswas et al. (1962), namely about $4 \times 10^{7} \mathrm{He} / \mathrm{cm}^{2}$. Because of these uncertainties, it seems best not to state a $\mathrm{He}^{3} / \mathrm{He}^{4}$ ratio at this time. 


\section{ELECTRONS}

The abundance of very energetic electrons is expected to be small due to the high rate of energy loss by synchrotron radiation at large relativistic energies; so, even if there were large numbers of these energetic electrons initially, they would soon lose their energy.

Although the flux of very energetic solar electrons is known to be relatively small, appreciably less than the proton component, positive evidence for electrons does exist for at least one event (Meyer and Vogt, 1962). In the July 18, 1961 event, the flux of electrons greater than $100 \mathrm{Mev}$ was measured to be $0.04 \mathrm{el} /\left(\mathrm{cm}^{2} \mathrm{ster} \mathrm{sec}\right)$, or a few percent of the proton flux in the same kinetic energy region. In other events, September 3, 1960 (Meyer and Vogt, 1962; Earl, 1962), November 12, 1960 (Yates, 1963), and November 15, 1960 (Ney and Stein, 1962), and March 16, 1964 (C1ine, Ludwig and McDonald, 1964), there was no positive of electrons. On September 4, 1960, Earl (1962) obtained an upper limit of 0.0025 for the rigidity interval greater than 450 Mev/c; the other limits in a similar rigidity range are less severe. During the March 16, 1964 solar event the 3 to 8 Mev electron flux increased less than $50 \%$ while the 15 to $75 \mathrm{Mev}$ proton flux increased by several orders of magnitude (Cline et al., 1964). Considering the flux levels and the general shape of the spectra, these results suggest an electron to proton ratio at about $5 \mathrm{Mev}$ of much less than $1 \%$.

In addition to the experiments related to direct measurements at the earth, Stein and Ney (1963) have shown that the continum electromagnetic radiation from large solar flares is consistent with the 
interpretation that this radiation is due to the synchrotron radiation of electrons. They have further shown that the number of electrons needed to produce the observed intensity is of the same order as the estimated number of protons accelerated in a large solar flare and that the necessary magnetic field is of the order of 500 gauss, which seems to be a conservative estimate of typical field strengths (A1ler, 1953). Therefore, present indications suggest that in fact the electrons are accelerated at the sun along with the protons, but lose their energy quickly by synchrotron radiation so that only a relativeiy few high energy electrons ever reach the earth.

Relatively large fluxes of low energy electrons (kinetic energy $>1 \mathrm{Mev}$ ) have also been observed. Hoffman et al. (1962) detected $3 \times 10^{6} \mathrm{el} /\left(\mathrm{cm}^{2}\right.$ ster $\left.\mathrm{sec}\right)$ between 10 and $35 \mathrm{kev}$. for about ten minutes on September 30,1961 , associated approximately with the arrival of the sudden commencement. Except for that period the flux was less than the detectable limit of $2 \times 10^{5} \mathrm{el}\left(\mathrm{cm}^{2}\right.$ ster sec).

E. NEUTRONS

No positive evidence is available at present for neutrons of solar origin. Anderson et al. (1959) obtained an upper limit of 0.02 for the ratio of neutrons above $120 \mathrm{Mev}$ to protons above $100 \mathrm{Mev}$ in a study during the August 22, 1958 solar particle event. Energetic neutrons are not particularly expected, because, being uncharged, the most likely acceleration mechanisms will have no effect on them. However, the need for more experiments in this area is clearly evident.

The possibility of solar neutrons from secondary processes during relatively quiet solar periods has been suggested in the literature. 
This question arose in connection with the apparent observation of a continuous quiet time flux of protons of energy less than $\sim 200 \mathrm{Mev}$ which was steady for a time period of several weeks during the years 1960 and 1961 when solar activity was relatively high (Meyer and Vogt, 1963a; and Stone, 1964). Satellite experiments seem to indicate that in 1961 the differential flux was almost constant from about 10 to $130 \mathrm{Mev}$ (Stone, 1964), and the results of Meyer and Vogt suggested the possibility of a rising spectra at low energies. In order to explain this phenomena, Simpson (1963) suggested that these low energy protons could be decay products of neutrons emitted from the sun. These solar neutrons might be produced, if protons and He particles were accelerated more or less continuously and suffered collisions deep in the solar atmosphere. The neutron flux expected on the basis of this hypothesis has been calculated under various assumptions (Lingenfelter and Flamm, 1964). However, there is strong evidence resulting from nuclear emulsion experiments flown on balloons to indicate that in the years 1960 and 1961 there was not a continuous quiet time flux (Freier and Waddington, 1965; Fichtel, Guss, Stevenson, and Waddington, 1964), and further there has been a systematic difference, where comparisons are available between the results of Meyer and Vogt and other experimentalists in the years 1962 through 1964 (Brunstein, 1964; Fichte1, Guss, Kniffen, and Neelakantan, 1964; Balasubrahmanyan, and McDonald, 1964; Freier and Waddington, 1964a; Ormes and Webber, 1964; Fichtel, Guss, and Durgaprasad, 1965; McDonald and Ludwig, 1964; Meyer and Vogt, 1963b). 
III. RELATIONSHIP OF THE COMPOSITION OF SOLAR COSMIC RAYS TO OTHER ASTROPHYSICAL PROBLEMS

In this section, the significance of the experimental data will be discussed in terms of four related problems. The first of these is the study of the propagation of solar particles in interplanetary space and the bearing of the composition data on this problem. Then, going back a step farther in the solar particles' history, a look will be taken at the acceleration of high energy solar particles. Thirdly, the relationship of the solar cosmic ray composition to that of the sun will be examined. Finally, there will be a discussion of the difference between the composition of the solar and galactic cosmic rays in detail in order to try to understand the importance of these differences in terms of the particles' origin and history.

\section{A. PROPOGATION OF SOLAR PARTICLES}

Beginning now with the propagation, there are two aspects which will be given some attention. The first is the type of propagation which occurs and the second is the amount of material which the particles have traversed before reaching the earth.

With regard to the former, it is realized now that the propagation of solar particles is not a simple matter. The function which describes the rise and fall of the particle intensity and the energy spectrum in principle, can depend on the particle velocity, rigidity, positron, and direction. Since the multiply charged particles have a different charge to mass ratio by a factor of two from that of the protons, they also have a very different rigidity from that of the protons for a given velocity. Thus, if the absolute differential energy spectra of 
both protons and helium nuclei, for example, could be measured throughout an event, together with their angular distribution, very significant insight could be obtained into the propagation characteristics because comparisons of fluxes at the same time could be made rather than having to compare fluxes of different velocities at different times as is the case of singly charged particles. In the last case, variations of the interplanetary conditions as a function of time can further complicate the picture. Unfortunately, only a few simultaneous measurements of the spectra of the two types of particles exist.

Even with these limited data, however, it seems clear that a purely rigidity dependent propagation can be excluded. As was shown earlier, the proton to helium ratio in the same rigidity intervals has been seen to vary appreciably from one time in a given event to another. Further, this ratio has varied very markedly from one event to another, while the helium to medium ratio has been the same in each of the three events it was measured.

Therefore, other general classes of propagation besides the pure rigidity one must be examined. One approach to the problem was the development of a simple diffusion model for solar cosmic rays wherein the scattering centers were magnetic inhomogenieties (Parker, 1956; Meyer, Parker, and Simpson, 1956; Parker, 1965 b). Although the diffusion coefficient in general can depend on both the particle's velocity and rigidity, Parker suggests that the propagation depends primarily on its velocity below $1 \mathrm{Bev}$ in the inner solar system because in this region the radius of gyration is less than the scale length of the 
magnetic field disordering. Considerable experimental evidence has now been accumulated to indicate that the time history of the energy spectrum does follow approximately the predictions of simple diffusion equations (Bryant, Cline, Desai, and McDonald, 1962; Hoffman and Winckler, 1963; Bryant, Cline, Desai and McDonald, 1965; Krimigis, 1965) and that at least early in an event the propogation is primarily velocity dependent (Bryant et al.,1962; Biswas et a1., 1962; Bryant et al.,1965).

During the declining phase, a somewhat different picture appears. The fact that an exponential decay of particle flux is often, or usually, seen late in an event (Webber, 1964; Bryant et al., 1962) suggests that particles are escaping from the region in which they are being held (Parker, 1962). It is unlikely that this boundary is absolutely sharp, but rather consists of a volume in which the magnetic field irregularities are less frequent and weaker.

However, the primarily garden hose nature of the magnetic field which has now been measured (Ness, Scearce, and Seek, 1964) cannot be ignored, and the diffusion theory must take into account the superimposed effects of magnetic irregularities and the semiregular fields. Theoretical calculations aimed at beginning to solve this problem have been made (Fibish and Abraham, 1965). One interesting result is the conclusion that the time history expected from other field configurations may bear many similarities to simple diffusion (Boldt, 1964). The anistropy, however, can be very different. 
Turning now to the amount of material traversed, there are a number of ways in which this parameter could be determined from the composition. These methods consist, for the most part, in measuring the abundances of some isotope or charge species which presumably exist in only a negligible amount on the sun, but is formed by fragmentation in the acceleration region or during the interplanetary propagation. Other less quantitative means of estimating the material traversed are related to the study of the energy spectra and relative abundances of various components. In the next several paragraphs the estimates of the amount of material traversed using severai different approaches will be discussed.

The upper limit to the relative abundances of $\mathrm{Be}$ and $\mathrm{B}$ nuclei given in Table 5 sets a limit on the number of these nuclei which could have been formed by the fragmentation of $\mathrm{S}$ nuclei (nuclear charge 26 ). In accordance with the available data on cross sections and the anaiysis used to supplement the existing data presented by Badhwar, Daniel, and Vijagalakshmi (1962) and Badhwar and Daniel (1963), the cross-sections for producing $\mathrm{Be}$ and $\mathrm{B}$ from $\mathrm{C}, \mathrm{N}, 0$, and $\mathrm{Ne}$ at 55 Mevinucleon (the mean of the experimental results) are 320,133, 100, and $40 \mathrm{mb}$ respectively. The uncertainties of these values could be as high as a factor of about two. The contribution from $z \geq 10$ nuclei is smail. Using the relative abundances of solar particle radiation as given in Tabie 5, the above cross-section values, and the observed 1 imit of the abundance of $\mathrm{Be}$ and $\mathrm{B}$, the amount of matter traversed is found to be $<0.1 \mathrm{gm} / \mathrm{cm}^{2}$ of $\mathrm{H}$. Assuming the uncertainty of the cross-section 
to be a factor of two, the limit becomes $<0.2 \mathrm{gm} / \mathrm{cm}^{2}$ of hydrogen.

Tritons are another particle species which must be formed primarily by fragmentation. Using the cross-section data assembled by Tilles et al. (1963) and Flamm et al. (1962) and a H to He ratio of 20, which seems typical for the energy interval of interest, the limit to the $\mathrm{H}^{3} / \mathrm{H}^{1}$ ratio obtained earlier, namely $<10^{-3}$, gives an upper 1 imit to the amount of material traversed of $0.5 \mathrm{gm} / \mathrm{cm}^{2}$. In this calculation, $\sigma\left[\mathrm{He}^{4}(p, d) \mathrm{H}^{3}\right]$ was assumed to be $30 \mathrm{mb}$.

In the events where only an upper limit could be set to the deutron flux the results are similar, and, in the two events where a small deuteron flux was observed, the calculated amount of material would be about the same as the upper limit in the other events or a little larger. However, this approach may not be too meaningful for the events in which McDonald et al. (1965) detected deuterons because the upper limit to the helium flux is so $\operatorname{small}\left(\mathrm{H}^{1} / \mathrm{He}^{4}>200\right)$ that it seems very unlikely that deuterons were formed by $\mathrm{He}^{4}$ fragmentation, unless the helium nuclei are subsequently suppressed for some reason. The ratio of $\mathrm{He}^{3} / \mathrm{He}^{4}$ in the solar beam is somewhat uncertain as previously discussed. However, if the accelerated solar particles traversed $<0.1 \mathrm{gm} / \mathrm{cm}^{2}$ of hydrogen, the cross-section data of Tilles et al. (1963) and Flamm et al. (1963) lead to a $\mathrm{He}^{3} / \mathrm{He}^{4}$ value of $<3 \times 10^{-3}$. This value is about 70 times smaller than the reported value of 0.2 , which had an uncertainty of a factor of three. The observed value gives the amount of matter traversed as $6-4^{+12}$, which is inconsistent with other observations in the November 12, 1960 event. 
However, as Fireman (1963) points out, it may also be possible that the $\mathrm{He}^{3}$ were formed in thermonuclear reactions as suggested by Severniji and Shabanskii(1960). Energetic $\mathrm{H}^{3}$ and $\mathrm{He}^{3}$ can be produced in this way, but not $\mathrm{H}^{2}$ nuclei which are the thermal energy parent particles.

There is other evidence which argues strongly against the amount of material traversed being as large as several $\mathrm{gm} / \mathrm{cm}^{2}$ in the large events. First, if the particles had passed through an amount of material approaching several $\mathrm{gm} / \mathrm{cm}^{2}$, the source energy/nucleon spectrum of the medium nuclei would have to be substantially different from that of the helium nuclei with a very much steeper spectrum in the vicinity of 100 to $200 \mathrm{Mev} /$ nucleon. Further, the $M /(\mathrm{z} \geq 10)$ ratio would be greater than the expected solar ratio rather than equal to it. It seems more reasonable to assume that the observed composition and energy spectra are a result of an unbiased acceleration of all nuclei rather than the coincidence of a biased acceleration and a just properly compensating amoung of material traversed before reaching the earth. Finally, observations of solar proton spectra in the energy interval of about 2 to $80 \mathrm{Mev}$ by detectors in satellites (Bryant, Cline, Desai, and McDonald, 1965) during several events do not show any flattening or decline of the proton spectrum below about $50 \mathrm{Mev}$, as would have occurred if the radiation had a smooth spectrum at the source and traversed a few $\mathrm{gm} / \mathrm{cm}^{2}$ of hydrogen. Bryant et al. (1965), in fact, suggest that the amount of material traversed may be less than .001 $\mathrm{gm} / \mathrm{cm}^{2}$ from studies of the proton energy spectrum. 
If it is postulated that the amount of material traversed, after the acceleration phase has begun is less than a few tenths of a $\mathrm{gm} / \mathrm{cm}^{2}$ then this amount of material is not sufficient to affect the relative abundances of the multiply charged nuclei in the measured energy interval by a detectable amount. Further, it was shown earlier in this section that the relative abundances of particles with the same charge to mass ratio in a given velocity interval will remain unaffected by the propagation since they have the same velocity for a given rigidity. The constancy of the helium to medium ratio both with time and energy and the contrastingly large variations in the proton to helium ratio then strongly suggest that the medium and helium nuclei have the same ionized charge to mass ratio. The only reasonable conclusion then is that these nuclei are fully ionized, since it is not likely that oxygen nuclei would have four electrons removed and helium only one, for example. We are then led to the conclusion that the constancy of the relative abundances of the multiply charged component exists after the acceleration phase at the sun and before propagation to the earth.

The interesting question which immediately presents itself is whether or not the relative abundances of the multiply charged nuclei reflect those at the sun. This will be discussed after the next section's treatment on acceleration phenomena.

It should also be mentioned that if there is interplanetary acceleration, which presently seems to be small compared to the initial acceleration, it probably has the same characteristics as the acceleration at 
the sun, which will be discussed in the next section. Therefore, it will have no effect on the above discussion of the constant ratios.

\section{B. ACCELERATION OF SOLAR PARTICLES}

The problem of the solar acceleration of particles to high energies is one that is far from solved. Various possible acceleration mechanisms have been examined in some detail previously (Parker, 1957; Parker, 1958; Alfven, 1959; Freier and Webber, 1963), and it has been concluded that there are three accelerating mechanisms which seem to be possibilities. They are the Fermi mechanism (Fermi, 1949; Fermi, 1954; Parker, 1958), the betatron effect (Alfven, 1959), and the magnetic field annihilation method including associated electric fields (Sweet, 1958; Dungey, 1958). Each of these three in turn permits a large number of variations depending on the individual model selected. In addition, there may be important special injection mechanisms, such as thermonulear reactions (Severnji and Shobanskii, 1960).

The Fermi mechanism is divided into two phases, injection and acceleration which is achieved by particles being reflected from moving magnetic inhomogeneities. Since the energy necessary for injection in a given physical situation can be a function of the charge and atomic weight of the nucleus, it is possible to have a biased composition; however, if the effective temperature is such that the mean particle energy is greater than that necessary for injection, no bias will occur. The nuclei may be ionized in other ways, for example by high energy electrons; however, the expected electron density makes this latter possibility seem unlikely. In the subsequent accele- 
ration, the first order Fermi theory leads to a particle spectrum which is a function of velocity only, regardless of its charge to mass ratioit being only necessary that the particle be charged. Previously it has been shown (Biswas, Fichtel, and Guss, 1962) that in a particular situation such as the sun one can obtain a spectrum which is both a function of velocity and rigidity, with a particle having a smaller charge to mass ratio having a steeper energy spectrum at high energies. This modified Fermi mechanism would lead to similar spectra for all the nuclei with the same charge to mass ratio but different spectra for protons and helium nuclei, as observed.

The study of the problem of acceleration of cosmic rays has also been investigated by Hayakawa, Nishimura, Obayashi, and Sato (1965). For solar cosmic rays, they conclude that the Fermi process predominates for the acceleration of nuclear particles in the non-relativistic region. The injection energy is assumed to be $10^{3}$ ev corresponding to a thermal energy for which $\mathrm{T}=10^{7} \mathrm{o}$, which is large, but possibly present in the source region. The energy gain then proceeds in the normal Fermi manner by the particles having collisions with magnetic irregularities moving with Alfven velocities, and the rate of change of energy is proportional to the total energy. This process ends when the particle escapes from the accelerating region. Their calculation shows that the final rigidity is proportional to $M / Z$ and is therefore the same for all multiply charged nuclei, but is different for protons and helium nuciei, for example. Their theory is also consistent with an exponential spectrum at low rigidities. 
A number of attempts have been made to find an astrophysical situation in which betatron acceleration can occur. Parker (1958) suggests that realistic calculations for sunspot regions indicate that the betatron effect by itself is not an important process because the energy gained is too small and the process is reversible. Even, if one includes a scattering effect to partially eliminate the complete reversibility, as Alfven (1959) has, the betatron effect still does not seem too likely because of the necessity of having to go through several cycles of very large scale magnetic field fluctuations in a relatively short time.

The magnetic field annihilation-electric field acceleration method has not been pursued very far generally because of the difficulty in understanding how it would be possible to obtain the necessary acceleration in a region whose physical parameters are similar to those which the photosphere, the chromosphere, or even the corona are thought to have. An excellent discussion of this problem is given by Parker (1963a)

Recently, McDonald et al. (1965) have suggested the possibility of a two-phase acceleration, in which the particles gain some energy while passing through a considerable amount of material in the first phase and then pass through relatively littie during the final phase. This type of process would explain the presence of $\mathrm{H}^{2}, \mathrm{H}^{3}$ and $\mathrm{He}^{3}$ and yet permit a smooth steep final proton spectrum and similar heavy nuclei spectra. The possible variation in composition among $H^{2}, H^{R}$, $\mathrm{H}^{3}$ and $\mathrm{He}^{4}$ suggests there may be important differences in the 
acceleration processes in different events. A complete picture remains to be developed, but these ideas should perhaps be explored further.

In general, the study of the multiply charged nuclei can aid greatly in the examination of the acceleration problem because it provides some definite facts which any proposed mechanism must explain. The results on $H^{3}, H^{\beta}$, and $\mathrm{He}^{3}$ must certainly be accounted for. Further, since the discussion in the preceding section indicated that the propagation mechanism probably would not affect the relative abundances of the multiply charged nuclei within a given velocity interval, the following features related to the observed characteristics then also apply to the particles immediately after acceleration. Firstly, the multiply charged nuclei have had the same relative abundances in the same velocity intervals within uncertainties each time a measurement was made, whereas this has not been true for the protons and heilum nuclei which have different charge to mass ratios. Secondly, insofar as comparisons can be made this composition reflects that of the sun. Thirdly, the helium and medium nuclei have had the same energy per nucleon spectrum in the two events where measurements could be made at the same time, whereas the proton and helium nuclei spectra in the same events were very different.

These results place important restraints on any acceleration theory and should aid in determining the true accelerating mechanism. For a further discussion of the solar particle acceleration problem, 
besides the references already mentioned see Dungey (1964), Gold (1964), Jaggi (1964), Parker (1957), Parker (1964), Petschek (1964), Severney (1960), Sweet (1964), and Wentzel (1964).

Finally, a tentative estimate of the time of acceleration may be obtained in the following manner. Assume that the solar cosmic rays have not passed through more than $0.1 \mathrm{gm} / \mathrm{cm}^{2}$, i.e., $6 \times 10^{22}$ atoms of hydrogen $/ \mathrm{cm}^{2}$, during the final acceleration phase as indicated by some of the data presented earlier. Since the amount of material traversed in interplanetary space is negligible compared to that in the solar atmosphere, this limit for the materiai traversed applies to the solar region. The density of matter at the flare site is poorly known, but has been estimated as being $10^{11}-10^{13}$ atoms of hydrogen/. $\mathrm{cm}^{3}$ (Gold and Hoyle, 1960; and Parker, 1957). A particle with an average energy between 10 and $50 \mathrm{Mev}$, nucleon has a velocity between $0.4 \times 10^{10}$ and $1.0 \times 10^{10} \mathrm{~cm} / \mathrm{sec}$. Hence, the acceleration time with these assumptions is not more than a few minutes and probably iess. On the other hand if the amount of material traversed were of the order of $3 \mathrm{gm}, \mathrm{cm}^{2}$, the time would be in the range from .3 to 70 minutes. However, since the early phase of the acceleration may occur in the more dense regions, the lower limit may be closer to the true value.

\section{RELATION TO SOLAR COMPOSITION}

There are a number of pieces of experimental data which suggest that the abundances of the multiply charged nuclei in the range $(2 \leq Z \leq 20)$ reflect those of the sun. Before processing further, these features will be summarized. First, the experimentally observed 
values for the relative abundances of the multiply charged energetic solar particles were the same as those obtained by spectroscopic means for those nuclei where a comparison could be made, namely carbon, nitrogen, oxygen, and some of the larger nuclei, and the upper limit of the Be and B flux was certainly consistent. The results show further that within the uncertainties the same relative abundances of helium, carbon, oxygen, neon, and larger nuclei have been obtained in a total of five exposures in two different events. Also, in a third event, when the fluxes were very small, at least the ratio of helium to medium nuclei was the same. Next, the helium nuclei are seen to have the same energy per nucleon, or velocity, spectrum as the medium nuclei at five times in two different events, every case when a measurement could be made. This is very good evidence to support the contention that they also have similar velocity spectra at the source. This last result together with the very different velocity spectra for protons suggest that the helium and medium nuclei were affected in the same way by the acceleration process.

Thus, the energetic solar nuclei coming from the sun with charges ranging from that of helium through at least about twenty do seem to reflect the composition of the solar surface. If the composition of these nuclei is accepted as representative of the sun, the relative abundances given in Table 5 may be used to estimate the helium and neon abundances in the sun, whereas it is not possible to obtain a good estimate of the abundance of these two elements spectroscopicaily in the photosphere. The average helium to oxygen ratio is $107 \pm 14$ and 
the average neon to oxygen ratio is $0.13+0.02$ (Biswas and Fichtel, 1963). The neon to oxygen ratio is similar to the universal abundances estimated by Suess and Urey (1956) and Cameron (1959), although a bit on the low side. The helium to medium ratio is typical, but of course, the more interesting ratio would be the proton to helium one. For the reasons mentioned above associated with the different energy spectra and charge to mass ratio, there is no simple reliable way to determine this ratio from solar cosmic rays alone. If the helium to medium ratio of $60 \pm 7$ is accepted as representative of the sun and the proton to medium value from spectroscopic data, namely 650 (Aller, 1961; Goldberg, Muller, and Aller, 1960), is used, a proton to helium ratio of $11_{-5}^{+7}$ is obtained. The uncertainty in this number depends on the correctness of the assumption above and the uncertainty in the proton to medium ratio. Hence, the estimated error placed on this ratio is large.

It is beyond the intended scope of this review article to discuss the relationship of these abundance ratios to solar models. Sears (1964) has discussed this question in some detail, and the reader is referred to this article.

\section{RELATION TO GALACTIC COSMIC RAYS}

Until a few years ago, it was generally accepted that most stars could not be important sources of cosmic rays for a number of reasons. Within the last several years it had been noted that, since the sun produced large quantities of particles whose energies were well above the injection energy needed for the Fermi theory to be operative in 
the galaxy (Fermi, 1949; Fermi, 1954), it might be possible that ordinary stars were an important source of cosmic rays. Relatively generous estimates of particle production by the stars indicated that protons could be supplied at a rate sufficient to account for the ordinary cosmic rays, and these particles could then be accelerated in the galaxy by the Fermi method. There remained the problem of the charge composition of cosmic rays being different from normal stellar abundances, but it was thought that there might possibly be favorable acceleration of the larger nuclei.

The results of the experiments on the multiply-charged component have shown that their abundances are just a reflection of those in the sun. There are at least four important differences within the heavy nuclei group between solar cosmic rays and ordinary cosmic rays. Two of these, the carbon to oxygen ratio of $3 / 5$ in solar cosmic rays compared to $3 / 2$ in ordinary cosmic rays and the light to medium ratio of $<1 / 100$ in solar cosmic rays compared to $1 / 4$ in ordinary cosmic rays may be attributed to the fact that ordinary cosmic rays have gone through a few $\mathrm{gm} / \mathrm{cm}^{2}$ of material wherein the light nuclei are formed by fragmentation, and there is at least an increase in the carbon to oxygen ratio. Also relatively minor variation in the source composition may aid in explaining the inverse $c / O$ value. The other two, the different helium to medium nuclei ratios and the different ratios between the helium nuclei and those in the high charge group are only enhanced by fragmentation. The helium to medium nuclei ratio is more than four times larger for the accelerated solar particles, about 
$(60 \pm 7): 1$ as compared to $(14 \pm 2): 1$ for ordinary cosmic rays, and the ratio of the helium nuclei to those in the charge group with $11 \leq \mathrm{Z} \leq 18$ is $(803 \pm 93): 1$ in the solar cosmic rays as compared to $(48 \pm 7): 1$ in the galactic cosmic rays-a difference of $16 \pm 3$. Finally, the helium to iron nuclei ratio is $\leq 5 \times 10^{3}$ in the solar cosmic rays compared to $171 \pm 26$ in the galactic cosmic rays-a difference of more than 25 . At galactic cosmic ray injection energies, the proton to medium ratio in solar cosmic rays is seen to be approximately $1: 10^{3}$ or larger. The proton to medium ratio for ordinary cosmic rays is about 250 for the same energy per nucleon intervals at very high energies and 100 for the same rigidity intervals. Thus, the proton to medium ratio for solar cosmic rays is 4 to 10 times the ordinary cosmic ray ratio and the difference would be slightly increased by fragmentation in interstellar matter. The differences in the ratios of the proton flux to that of either the medium nuclei or heavier nuclei are much greater as can be seen by an examination of the ratios in the previous paragraph.

Thus, since the sun has abundances typical of most ordinary stars* in the respects mentioned here and since these abundances are reflected in solar cosmic rays, it seems reasonable to conclude that the difference in the charge composition between galactic cosmic rays and ordinary starsbeing considered as the sole primary source of galactic cosmic rays. Thus, galactic cosmic rays probably have a very special origin, perhaps in supernovae [See for example Ginzburg and Syroratsky (1961)].

\footnotetext{
*Here the term ordinary star refers to the great majority of stars with normal cosmic abundances and specifically excludes the unusual ones such as nova and supernovae which may be important cosmic ray sources
} 
The general features of the solar particle composition now seem to be clear. The two most abundant components, protons and helium nuclei, have different velocity spectra, similar, but not exactly identical rigidity spectra, and varying relative abundances. The multiply charged nuclei, on the other hand, appear to have the same spectral shape and relative abundances each time measurements are made, at least in the region from 42 to $135 \mathrm{Mev} /$ nucleon. Further, these relative abundances seem to reflect those of the sun insofar as comparison can be made. Electrons are rare, but high energy electrons are not expected to be plentiful due to the probable high rate of energy loss caused by synchrotron radiation at the sun. Energetic neutrons were also not expected in large quantity and have not been observed. Finally, there is positive evidence that very sma1l quantities of deutrons and tritons exist, probably in an amount which is about $10^{-3}$ or less of the proton abundance.

The experimental data indicate that the propogation phenomenon is not purely rigidity dependent. Although the propogation of solar particles is still not weli understood, the development of theories which take into account both the general magnetic field and the inhomogeneities in the field seem to hold some promise of explaining the experimental results. The composition data have also established important restraints which any acceleration theory must satisfy, and thereby contributed greatly to the very difficuit problem of determining the acceleration mechanism. 
- The similarity of the relative abundance of the energetic solar particles and the nuclei in the sun's photosphere suggested the possibility of having a new means of estimating the solar neon and helium abundances. This very interesting possibility will have to be explored by further testing of the composition of future solar particle events. Finally, it was seen that the composition was a very strong argument against most stars being the principal source of high energy non-solar cosmic rays, and, therefore, special sources, such as supernovae or possibly quasistellar objects, should be considered as much more likely prospects for the origin of cosmic rays.

The results which have been obtained thus far on the composition of solar cosmic rays have indicated that further research in this area of study should be very rewarding and of value to many fields of physics. Further data on the composition and relative, as well as absolute, energy spectra of the various components are needed throughout many events. More experiments also should be performed to determine the properties of the rare components, deuteron, tritons, $\mathrm{He}^{3}$, electrons, neutrons, and the heavier nuclei. When these experiments are complete, the knowledge which is needed to aid in answering the solar and astrophysical problems discussed in this review should be at hand. 
REFERENCES

Alfven: 1959, Tellus XI, 106

Aller, L. H: 1953, Astrophysics, The Atmospheres of the Sun and Stars, The Ronald Press Company, New York.

Anderson, K. A., R. Arnoldy, R. Hoffman, L. Peterson and J. R. Winckler: 1959, J. Geophys. Res. 64, 1133.

Anderson, K. A., and J. R. Winckler: 1962, J. Geophys. Res. 6ㅁ, 4103.

Badhwar, G. D., R. R. Daniel and B. Vijayalakshmi: 1962, Prog. Theo. Phys. 28, 607 .

Badhwar, G.D. and R. R. Danie1: 1963, Prog. Theo. Phys. (to be published).

Ba lasubrahmanyan, V.K. and F. B. McDona1d: 1964, J. Geophys. Res. $\underline{69}, 3289$.

Biswas, S: 1961, J. Geophys. Res. 67, 2613.

Biswas, S: 1963, Proc. International Conf. on Cosmic Rays, Jaipur $\underline{1}, 43$.

Biswas, S., and C. E. Fichtel: 1963, Astrophys. J. 139, 941.

Biswas, S., C. E. Fichtel and D. E. Guss: 1962, Phys. Rev. 128, 2756.

Biswas, S., C. E. Fichtel, D. E. Guss and C. J. Waddington: 1963,

J. of Geophys. Res., 68, 3109 .

Biswas, S., C. E. Fichtel and D. E. Guss: 1965, to be published.

Biswas, S., and P. S. Freier: 1961, J. Geophys. Res. 66, 1029.

Biswas, S., P. S. Freier, and W. Stein: 1962, J. Geophys. Res. 67, 13 .

Boldt, E., 1964: Goddard Space Flight Center Document \# X-611-64-371.

Brunstein, K. A.: 1964 , Phys. Rev. 133, B1520.

Bryant, D. A., T. L. Cline, U. D. Desai and F. B. McDonald: 1962 , J. Geophys. Res. 67, 4983 .

Bryant, D. A., T. L. Cline, U. D. Desai and F. B. McDonald: 1963, Proc. Internationa 1 Conf. on Cosmic Rays, Jaipur 1,70 .

Bryant, D. A., T. L. Cline, U. D. Desai and F. B. McDonald: 1965, Astrophys. Jour. $141,478$. 
Cameron, A. G. W.: 1959, Astrophys. Jour. $\underline{129}, 676$.

Chubb, T. A., H. Friedman and R. W. Kreplin: 1960, J. Geophys. Res. $\underline{65}, 1831$.

Cline, T. L., G. H. Ludwig and F. B. McDonald: 1964, Phys. Rev. Letters, $13,786$.

Davis, L. R., and K. W. Ogilvie: 1962, J. Geophys. Res. 67, 1711.

Dungey, J. W.: 1958, Cosmic Electrodynamics, Cambridge University Press, Cambridge, 125.

Dungey, J. W.: 1964, AAS-NASA Symposium of the Physics of Solar F1ares, US GPO, 415 .

Ear 1, J. A.: 1962, J. Geophys, Res. 67, 2107.

Fermi, E.: 1949, Phys. Rev. 75, 1169.

Fermi, E: 1954, Astrophys. Jour. 119,1 .

Fibish, M., and P. Abraham, "On the Propogation and Diffusion of Solar Particles", to be published in J. Geophys. Research.

Fichtel, C. E., and D. E. Guss: 1961, Phys. Rev. Letters $\underline{6}, 495$.

Fichtel, C. E., D. E. Guss, N. Durgaprasad, 1965: to be published.

Fichtel, C. E., D. E. Guss, D. A. Kniffen and K. A. Neelakantan: 1964 , J. Geophys. Res. 69, 3293 .

Fichtel, C. E., D. E. Guss, G. R. Stevenson, C. J. Waddington: 1964, Phys. Rev. 133, B818.

Fireman, E. L.: 1963, Proc. International Conf: on Cosmic Rays, Jaipur, $\underline{3}, 487$.

Fireman, E. L., J. DeFelice and D. Tilles: 1961, Phys. Rev. 123, 1935 .

Flamm, E., R. E. Lingenfelter, G. J. F. McDonald and W. F. Libby: 1962 , Science, 138,48 .

Forbush, S. E.: 1946, Phys. Rev., 70, 771.

Freier, P. S.: 1962 , J. Geophys. Res. 67, 2617.

Freier, P. S.: 1963, J. Geophys. Res. 68, 1805. 
Freier, P. S., and C. J. Waddington: 1964a, Phys. Rev. Letters, 13, 108.

Freier, P. S., and C. J. Waddington: 1964b, Phys. Rev. 136, B1535.

Freier, P. S., and C. J. Waddington: 1965, Private Communication

Freier, P. S., and W. R. Webber: 1963, J. Geophys. Res. 68, 1605.

Ginzburg, V. L. and A. I. Syrovatsky: 1961, Prog. Theo. Phys. Japan, Supp1. 20, 1 .

Gold, T.: 1964, AAS-NASA Symposium on the Physics of Solar Flares, US GPO, 389.

Gold, T., and F. Hoyle: 1960, Mon. Not. Roy. Astr. Soc. 120, 89.

Goldberg, L., E. A. Muller and L. H. Aller: 1960, Ap. J. Suppl. $\underline{5}$, 1 .

Hayakawa, S., J. Nishimura, H. Obayashi and H. Sato: 1965, "Accerlation Mechanisms of Cosmic Rays", Nagoya University preprint.

Hoffman, R. A., L. R. Davis and J. M. Williamson: 1962, J. Geophys. Res. 67, 5001.

Hoffman, D. J., and J. R. Winckler: 1963, J. Geophys. Res. 68, 2067.

Jaggi, R. N.: 1964, AAS-NASA Symposium on the Physics of Solar Flares, US GPO, 419.

Krimigis, S. M.: 1965, Midwest Cosmic Ray Conference, January.

Kurnosova, L. V., L. A. Razorenov, and M. I. Fradkin: 1962, J. Phys. Soc. of Japan, 17, Supp. A-II, 315.

Kurnosova, L. V., L. A. Razorenov and M. I. Fradkin: 1962, Zemli No. 12,31 .

La1, D., G. Rajagopalan, and V. S. Venkatavaradan: 1963, Proc. International Conf. on Cosmic Rays, Jaipur $\underline{1}, 99$.

Lingenfelter, R. E., and E. J. Flamm: 1964, Science 144, 292.

McDonald, F. B.: 1963, NASA TR R-169, with contributions by C. E. Fichtel, D. E. Guss, H. H. Malitson, K. G. McCracken, K. W. Ogilvie, and W. R. Webber. 
McDonald, F. B.: 1965, Private Communication.

McDonald, F. B., V. K. Balasubrahmanyan, K. A. Brunstein, D. E. Hagge, G. H. Ludwig and R. A. Palmeira: 1965, Transactions Am. Geophys. Un. $\underline{46}, 124$.

McDonald, F. B., and G. H. Ludwig: 1964, Phys. Rev. Letters, 13, 783. Meyer, P., E. N. Parker and J. A. Simpson: 1965, Phys. Rev. 104, 768. Meyer, P., and R. Vogt: 1962, Phys. Rev. Letters $\underline{8}, 387$.

Meyer, P., and R. Vogt: 1963a, Phys. Rev. 29, 2275.

Meyer, P., and R. Vogt: 1963b, Proc. International Conf. of Cosmic Rays, Jaipur, $\underline{3}, 49$.

Ness, N., C. Scearce and J. B. Seek: 1964, J. Geophys. Res. 69, 3531.

Ney, E. P., and W. Stein: 1962, J. Geophys. Res. 67, 2087.

Ogilvie, K. W., D. A. Bryant and L. R. Davis: 1962, J. Geophys. Res. $\underline{67}, 929$.

Ormes, J., and W. R. Webber: 1964, Phys. Rev. Letters 13, 106.

Parker, E. N.: 1956, Phys. Rev. 103, 1518.

Parker, E. N.: 1957a, Phys. Rev. 107, 830 .

Parker, E. N.: 1957b, J. Geophys. Res. 62, 509.

Parker, E. N.: 1958, Phys. Rev. 109, 1328.

Parker, E. N.: 1963a, Astrophys. J. Supp1. 77 to Vo1. VIII, 177.

Parker, E. N.: 1963b, Interplanetary Dynamical Processes, N.Y. Interscience Publishers.

Parker, E. N.: 1964, AAS-NASA Symposium on the Physics of Solar Flares, US GPO, 417.

Peterson, L. E., and J. R. Winckler: 1959, J. Geophys. Res. 697.

Petschek, H. E.: 1964, AAS-NASA Symposium on the Physics of Solar Flares, US GPO, 425 . 
Pomerantz, M. A., and L. Witten: 1962, Paper presented at the Third International Space Science Symposium of COSPAR, Washington, D.C., May.

Roederer; J. G.: 1964, Space Science Reviews III, 847.

Schaeffer, O.A., and J. Zahinger: 1962, Phys. Rev. Letters $\underline{8}, 389$.

Sears, R. L.: 1964, The Astrophysical Journal 140, 477.

Severney, A. B., and Shabanskii: 1960, Astrophysical Journal SSSR 37, 609 .

Simpson, J. A.: 1963, Pontificia Academia Scientiarium, Vatican, 323 .

Stein, W. A., and E. P. Ney: 1963, J. Geophys. Res. 68, 65.

Stone, E. C.: 1964, J. Geophys. Res. 69, 3939.

Suess, H. E., and H. C. Urey: 1956, Rev. Mod. Phys. 28, 53.

Sweet, P. A.: 1958, Nuovo Cimento Suppl. 8 Ser. X, 188.

Sweet, P. A.: 1964, AAS-NASA Symposium on the Physics of Solar Flares, US GPO, 409.

Tilles, D., J. DeFelice and E. L. Fireman: 1963, ICARUS, 2, 258. Waddington, C. J.: 1960, Prog. Nuclear Phys. $\underline{8}, 3$.

Waddington, C. J.: 1962, J. Phys. Soc. Japan, 17, Suppl. A-3, 63. Webber, W. R.: 1964, AAS-NASA Symposium on the Physics of Solar Flares, US GPO, 419 .

Wentzel, D. G.: 1964, AAS-NASA Symposium on the Physics of Solar Flares, US GPO, 397.

Winckler, J. R., T. C. May and A. J. Masley: 1961, J. Geophys. Res. 66,316 .

Yagoda, H. R. Filz and K. Fukui: 1961, Phys. Rev. Letters, 6, 626. Yates, G. K: 1963, Private communications of unpublished results. 


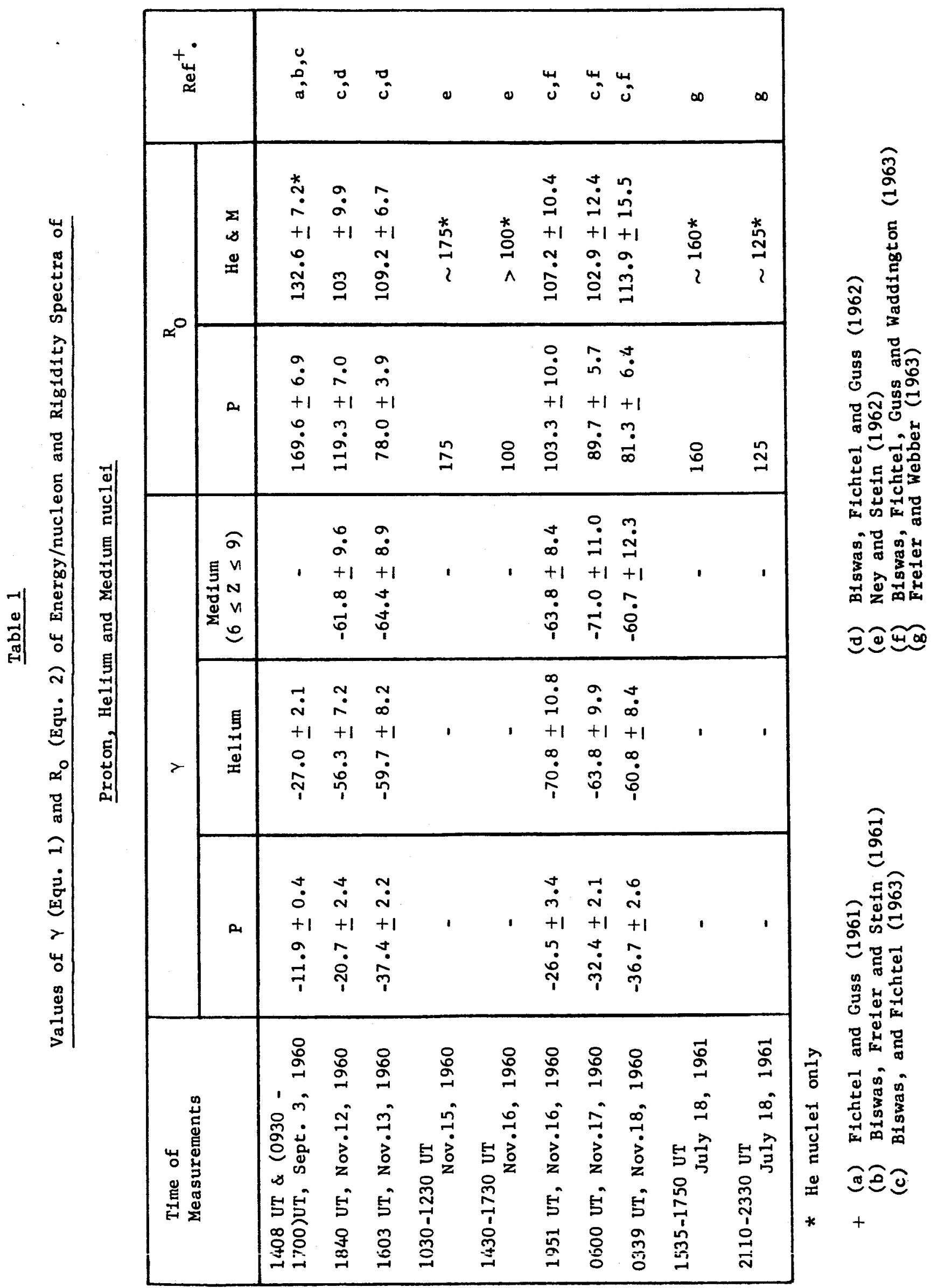




\begin{tabular}{|c|c|c|c|c|c|c|c|c|c|c|c|}
\hline \multirow{10}{*}{ 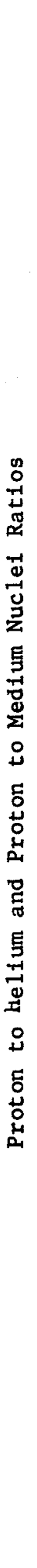 } & $\underset{\Perp}{凶}$ & $\stackrel{c}{a}$ & $\stackrel{-\varepsilon}{\infty}$ & $\ddot{\infty}$ & مـ & مـ & 4 & $\nabla$ & 7 & $\rightarrow$ & 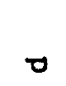 \\
\hline & 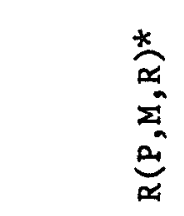 & ' & ' & I & 1 & ' & $\begin{array}{l}8 \\
0 \\
m \\
+1 \\
8 \\
0 \\
=\end{array}$ & $\begin{array}{l}\text { in } \\
+1 \\
8 \\
8\end{array}$ & $\begin{array}{c}\text { t⿱ } \\
* \\
\stackrel{8}{*} \\
i \\
i\end{array}$ & 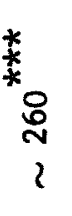 & $\begin{array}{l} \pm \\
+1 \\
+1 \\
\infty\end{array}$ \\
\hline & 宏 & $\begin{array}{l}1 \\
0 \\
+1 \\
+ \\
- \\
\end{array}$ & $\begin{array}{l}\infty \\
0 \\
+1 \\
0 \\
\dot{n} \\
\end{array}$ & $\begin{array}{l}\text { 은 } \\
N\end{array}$ & $\vec{m}$ & $\stackrel{9}{\rightarrow}$ & $\begin{array}{l}n \\
+1 \\
8\end{array}$ & $\begin{array}{l}-1 \\
+1 \\
\text { in }\end{array}$ & ' & ' & $\begin{array}{l}\Upsilon \\
0 \\
+1 \\
-1\end{array}$ \\
\hline & 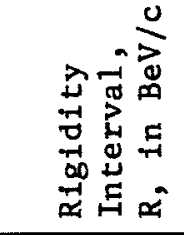 & $\begin{array}{l}O \\
\dot{0} \\
N\end{array}$ & $\begin{array}{l}9 \\
\vdots \\
0 \\
N\end{array}$ & $\begin{array}{l}0 \\
\dot{1} \\
\dot{0}\end{array}$ & $\begin{array}{l}\dot{0} \\
\dot{0} \\
\dot{0} \\
\dot{0}\end{array}$ & $\begin{array}{l}\dot{0} \\
\dot{1} \\
\dot{0} \\
\dot{0}\end{array}$ & 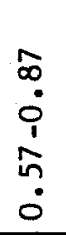 & $\begin{array}{l}\hat{0} \\
\dot{0} \\
1 \\
\hat{n} \\
\dot{0}\end{array}$ & $\begin{array}{l}n \\
i \\
i\end{array}$ & $\begin{array}{l}n \\
\dot{n} \\
\dot{n}\end{array}$ & $\begin{array}{l}\infty \\
\dot{0} \\
\dot{1} \\
\hat{n} \\
\dot{n} \\
\dot{0}\end{array}$ \\
\hline & 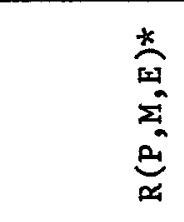 & 1 & 1 & 1 & 1 & 1 & 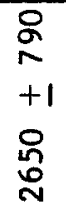 & $\begin{array}{l}8 \\
8 \\
+1 \\
\text { +1 } \\
8 \\
\text { 유 }\end{array}$ & ' & ' & $\begin{array}{l}\text { O } \\
\text { 子 } \\
+1 \\
\text { o } \\
\text { ֶั }\end{array}$ \\
\hline & 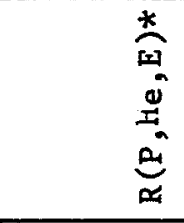 & $\begin{array}{l}0 \\
+1 \\
\text { in }\end{array}$ & $\begin{array}{l}0 \\
+1 \\
+0 \\
\stackrel{9}{子}\end{array}$ & 1 & 1 & $\begin{array}{l}\stackrel{n}{\infty} \\
\stackrel{0}{-1} \\
\wedge\end{array}$ & 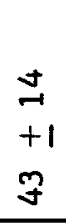 & $\begin{array}{l}0 \\
+1 \\
\tilde{m}\end{array}$ & ' & ' & $\begin{array}{l}n \\
+1 \\
0 \\
\end{array}$ \\
\hline & 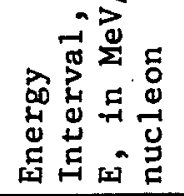 & $\begin{array}{l}\infty \\
N\end{array}$ & $\begin{array}{l}\infty \\
N \\
N\end{array}$ & ' & 1 & \begin{tabular}{l}
8 \\
$\infty$ \\
\multirow{1}{1}{} \\
$\stackrel{1}{7}$ \\
$=$
\end{tabular} & $\begin{array}{l}\stackrel{n}{a} \\
\dot{1} \\
\dot{\sim}\end{array}$ & $\begin{array}{l}\text { a } \\
\text { ஸे } \\
\text { ஸे }\end{array}$ & ' & I & $\begin{array}{l}2 \\
\dot{1} \\
\stackrel{\dot{\gamma}}{ }\end{array}$ \\
\hline & $\begin{array}{l}\stackrel{0}{J j} \\
\stackrel{-1}{\vec{J}} \\
\stackrel{0}{J}\end{array}$ & 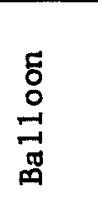 & 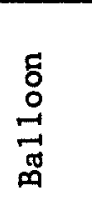 & 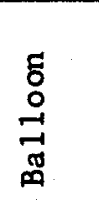 & 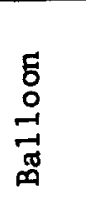 & 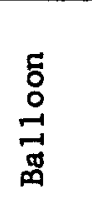 & 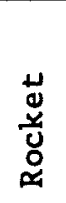 & 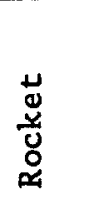 & 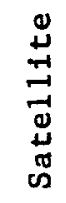 & $=$ & 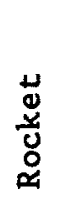 \\
\hline & 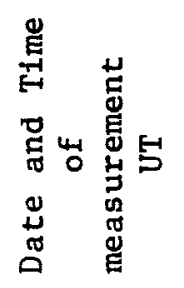 & 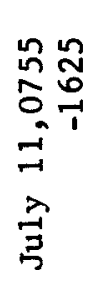 & 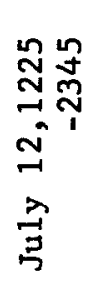 & 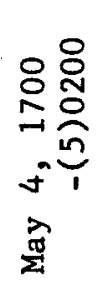 & 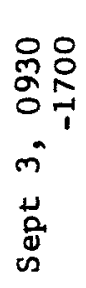 & 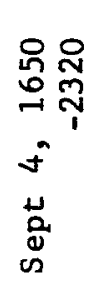 & 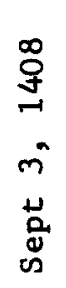 & 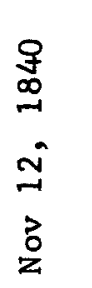 & 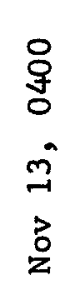 & 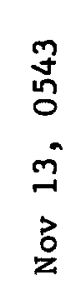 & $\begin{array}{l}0 \\
0 \\
\stackrel{0}{1} \\
m \\
2 \\
0 \\
0 \\
z\end{array}$ \\
\hline & 惫 & 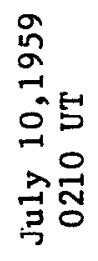 & & 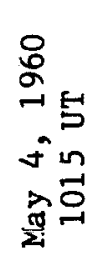 & 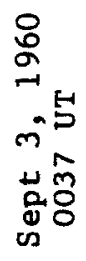 & & & 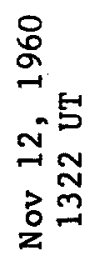 & & & \\
\hline
\end{tabular}




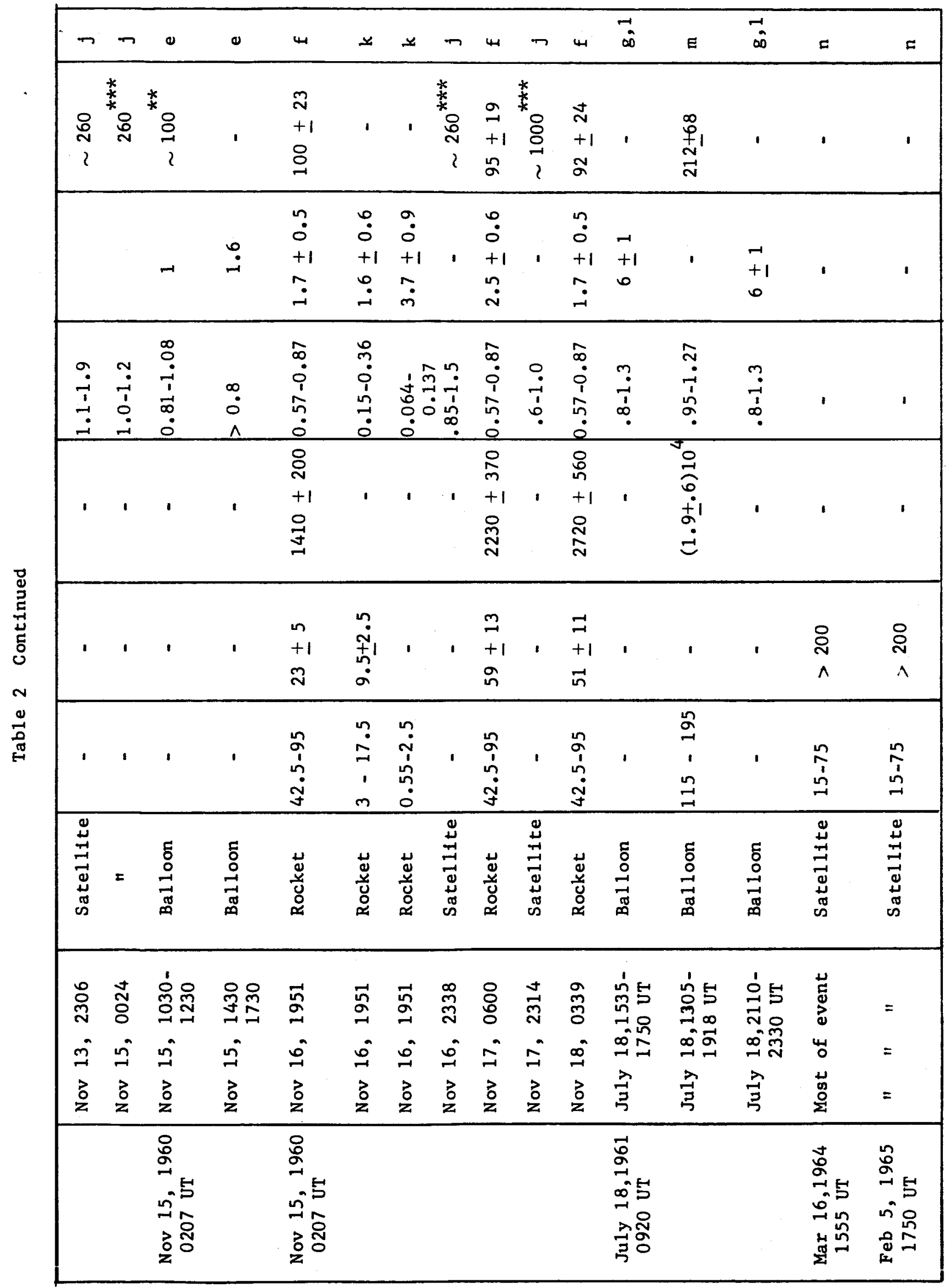




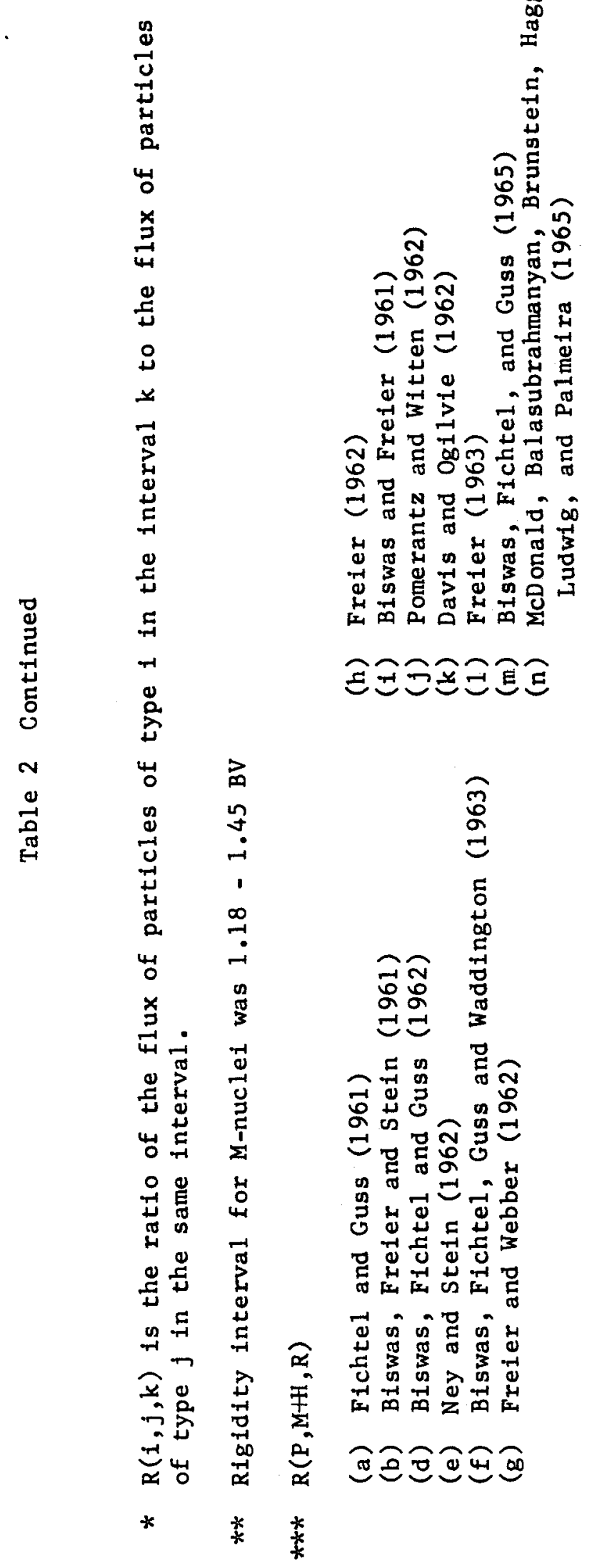


$\underline{\text { Table } 3}$

Helium to Medium Nuclei Ratio

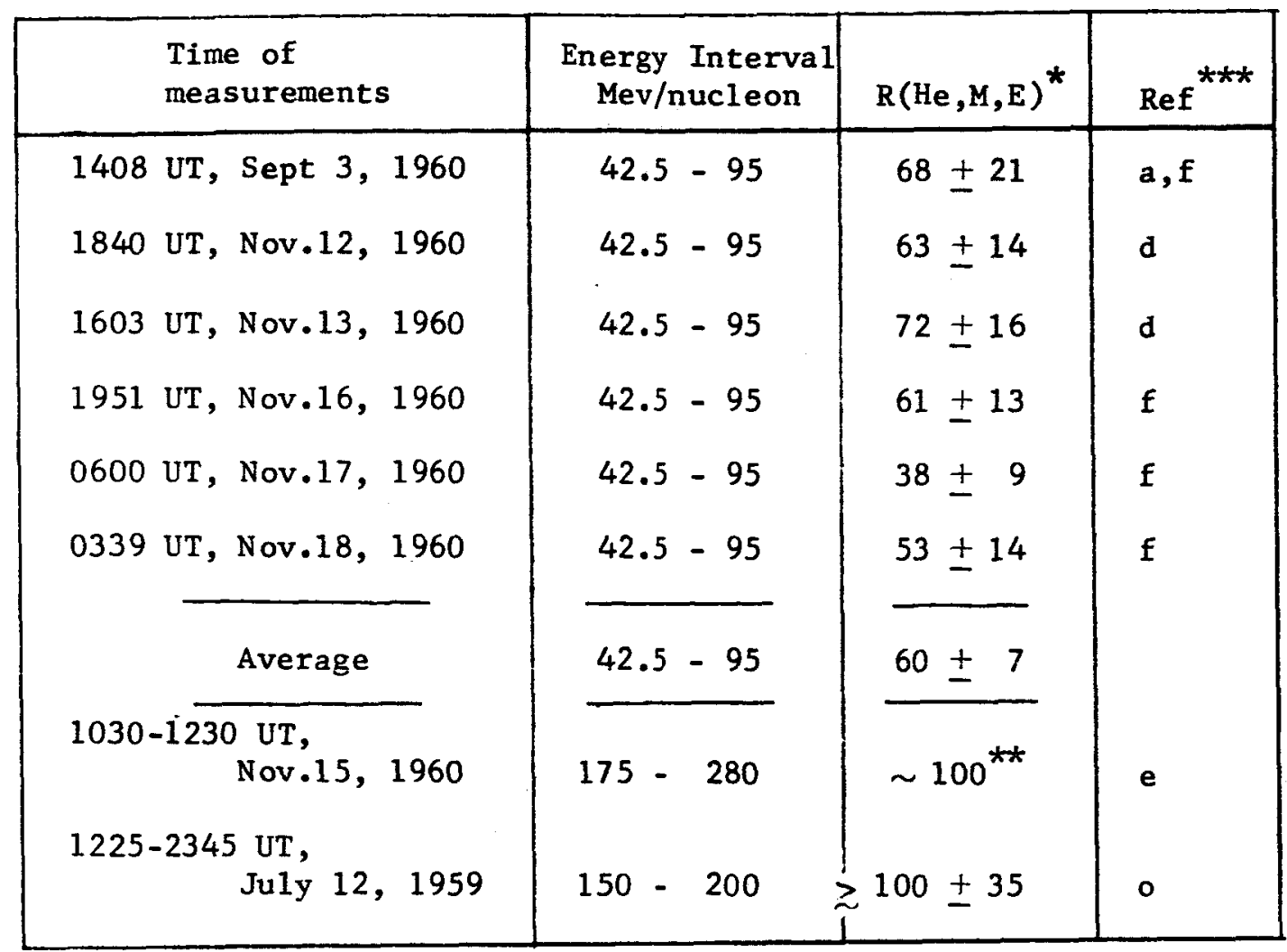

* $R(i, j, k)$ is the ratio of the flux of particles of type $i$ in the interval $k$ to the flux of particles of type $j$ in the same interval.

** Uncertainty of the value is a factor to two.

*** (a) Fichtel and Guss (1961)

(d) Biswas, Fichtel and Guss (1962)

(e) Ney and Stein (1962)

(f) Biswas, Fichtel, Guss and Waddington (1963)

(o) Biswas (1962) 


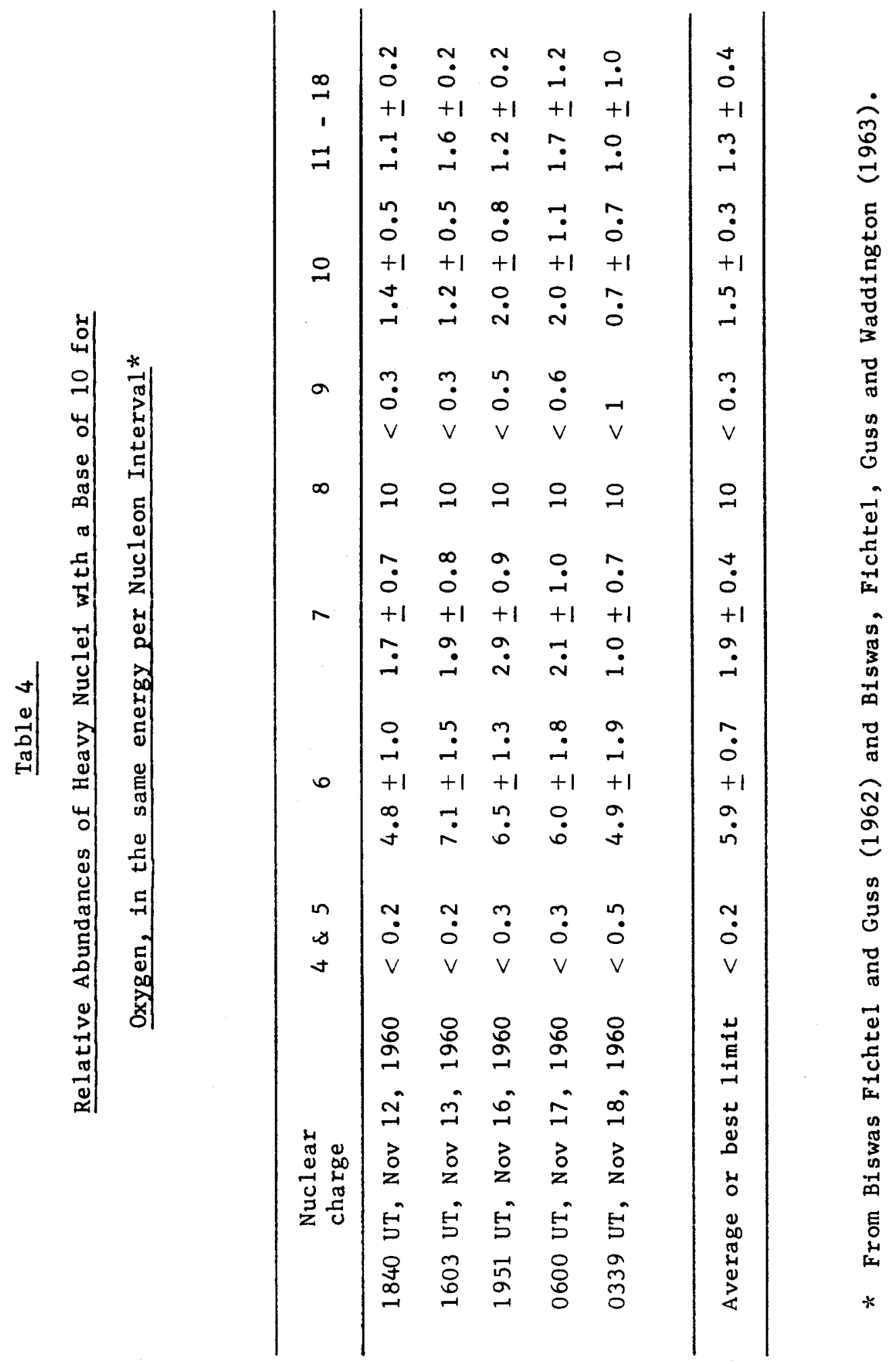


Table 5

Relative Abundances of Nuclei Normalized to a Base

of 1.0 for Oxygen

\begin{tabular}{|c|c|c|c|c|}
\hline Element & Solar Cosmic Rays & $\operatorname{Sun}^{+}$ & $\begin{array}{l}\text { Universa1 } \\
\text { Abundances }\end{array}$ & $\begin{array}{l}\text { Galactic Cosmic } \\
\text { Rayst* }\end{array}$ \\
\hline $2^{\mathrm{He}}$ & $107 \pm 14$ & $?$ & 150 & 48 \\
\hline $3^{L i}$ & - & $<10^{-5}$ & $<10^{-5}$ & 0.3 \\
\hline $4^{B e-} 5^{B}$ & $<0.02$ & $<10^{-5}$ & $<10^{-5}$ & 0.8 \\
\hline${ }_{6} \mathrm{C}$ & $0.59 \pm 0.07$ & 0.6 & 0.26 & 1.8 \\
\hline $7^{\mathrm{N}}$ & $0.19 \pm 0.04$ & 0.1 & 0.20 & $\lesssim 0.8$ \\
\hline $8^{0}$ & 1.0 & 1.0 & 1.0 & 1.0 \\
\hline${ }_{9} \mathbf{F}$ & $<0.03$ & 0.001 & $<10^{-4}$ & $\lesssim 0.1$ \\
\hline $10^{\mathrm{Ne}}$ & $0.13 \pm 0.02$ & $?$ & 0.36 & 0.30 \\
\hline $11^{\mathrm{Na}}$ & - & 0.002 & 0.002 & 0.19 \\
\hline $12^{\mathrm{Mg}}$ & $0.043 \pm 0.011$ & 0.027 & 0.040 & 0.32 \\
\hline $13^{\mathrm{A} 1}$ & - & 0.002 & 0.004 & 0.06 \\
\hline $14^{\mathrm{Si}}$ & $0.033 \pm 0.011$ & 0.035 & 0.045 & 0.12 \\
\hline $15^{P-21} S c$ & $0.057 \pm 0.017$ & $0.032^{* * *}$ & 0.024 & 0.13 \\
\hline $22^{\mathrm{T} i-}-28^{\mathrm{N} i}$ & $\lesssim 0.02$ & 0.006 & 0.033 & 0.28 \\
\hline
\end{tabular}

+ The uncertainty of the values in this column is probably of the order a factor of $0.5 \mathrm{sec}$. Aller (1961) or Goldberg, Muller, and Aller (1961)

+ The uncertainty of the values in this column is hard to estimate, but

+ is probably at least a factor of 0.5 in some cases. See Suess and Urey (1956) and Cameron (1959)

** The uncertainty of the values in this column varies from 10 to about 30 percent. See Waddington (1960)

*** A $5 / 2$ ratio for the abundance of $16^{\mathrm{S}}$ relative to $18^{\mathrm{A}}$ was assumed, the relative abundance of $18 \mathrm{~A}$ being unknown. 


\section{Captions for Figures}

Fig. 1. Differential rigidity spectra of solar protons, helium, and medium nuclei at 1951 UT, November 16, 1960 (Biswas, et al., 1963). 0: Protons; $\Delta$ : He Nuclei; 0: (Medium Nuclei) 60

Fig. 2. Differential rigidity spectra of solar protons, helium, and medium nuclei at 1603 UT, November 13, 1960 (Biswas and Fichtel, 1963〉 0: Protons; $\Delta$ : He Nuclei; 0: (Medium Nuclei)

60

Fig. 3. Differential energy spectra of solar protons, helium, and medium $(6 \leq Z \leq 9)$ nuclei as a function of total energy/rest energy at 1951 UT, November 16, 1960. Note that the abcissa is an expanded logarithmic scale (Biswas, et al., 1963). 0: Protons; $\Delta$ : He Nuclei 10; 1 : (Medium Nuclei) 600

Fig. 4. (a) Differential energy spectra for solar protons as a function of total energy for those instances where proton, helium, and medium spectra were observed simultaneously.
A - 1400 UT Sept. 3, $1960-\mathrm{O}-$
B - 1840 UT Nov. 12, $1960-\cdots-\Delta-\cdots$
C - 1603 UT Nov. $13,1960---\square-(-$
D - 1951 UT Nov. 15, $1960 \ldots \ldots$.
E - 0600 UT Nov. 16, $1960-\cdots-0-\cdots-$
F - 0339 UT Nov. 17, $1960, \cdots-$ 


\section{Captions for Figures (Continued)}

Fig. 4. (b). Differential energy spectra for solar helium and medium nuclei as a function of total energy for those instances where proton, helium, and medium spectra were observed simultaneously.

Time He M X 60

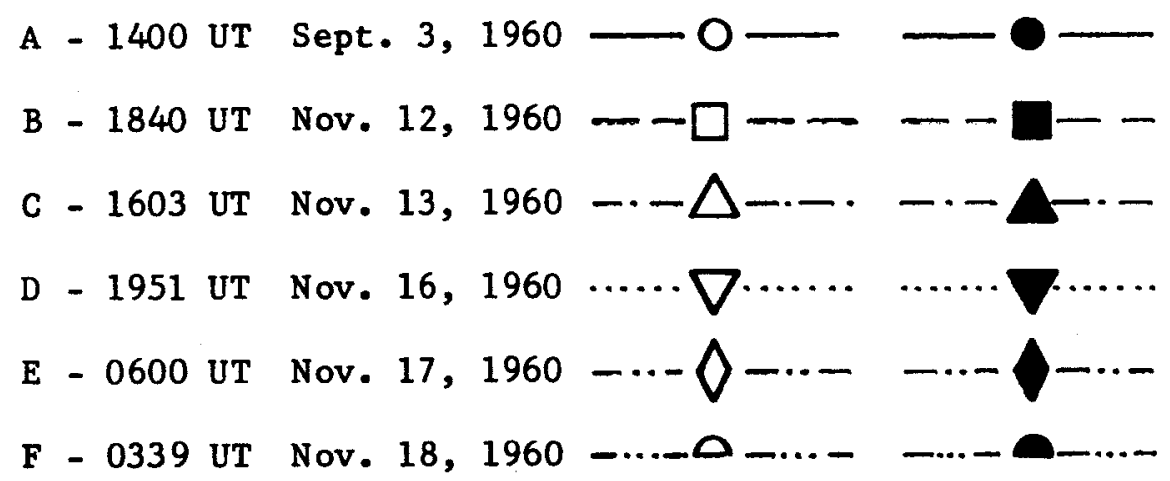

Fig. 5. The proton-to-helium nuclei ratio as a function of kinetic energy per nucleon at several different times. For curves A through F, the curves represent data taken from the work of Biswas, et al., (1962), Biswas, et-al., (1963), Biswas, (1961), and Biswas and Fichtel, (1963). Uncertainties in the ratios range from 25 to 50 per cent. The data represented by $G$ are the lower limits set by McDonald, et al., (1965). The times at which the measurements were made are as follows: A - 1840 UT, Nov. 12, 1960; B - 1603 UT, Nov. 13, 1960; C - 1961 UT, Nov. 16, 1960; D - 0600 UT, Nov. 17, 1960; E - 0339 UT, Nov. 18, 1960; F - 1408 UT, Sept. 3, 1960; G - March 16, 1964 and Feb. 5, 1965. 


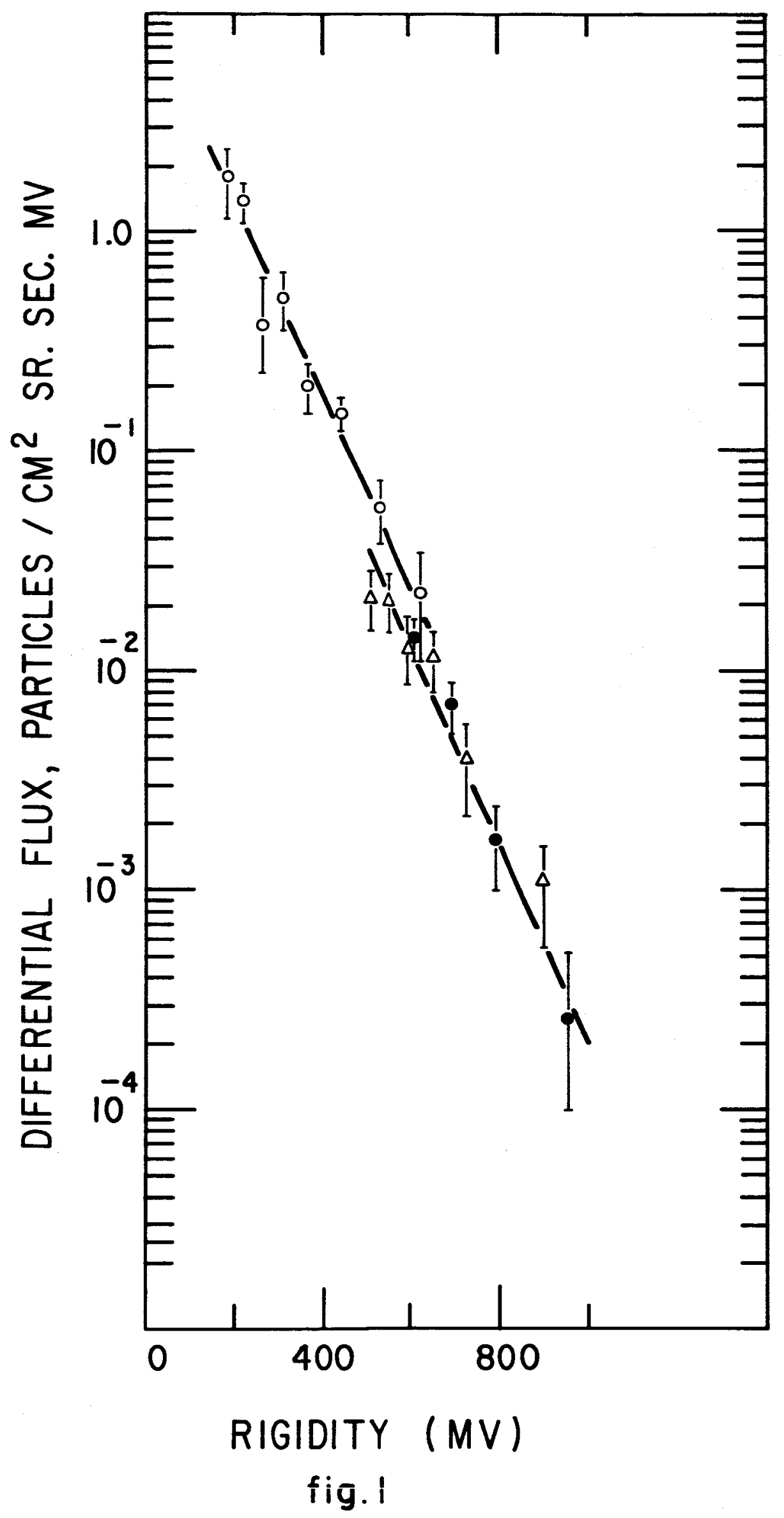




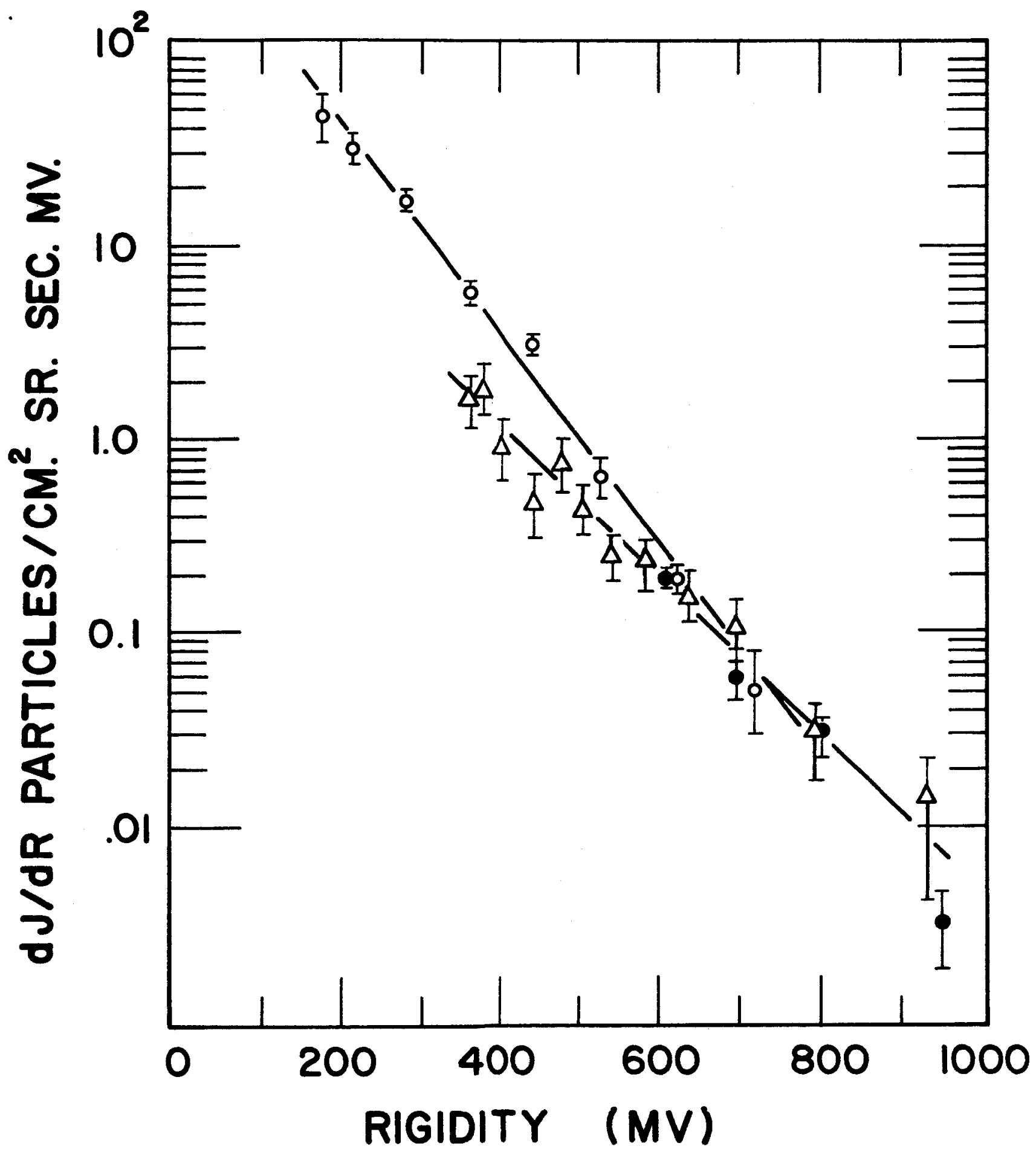

fig. 2 


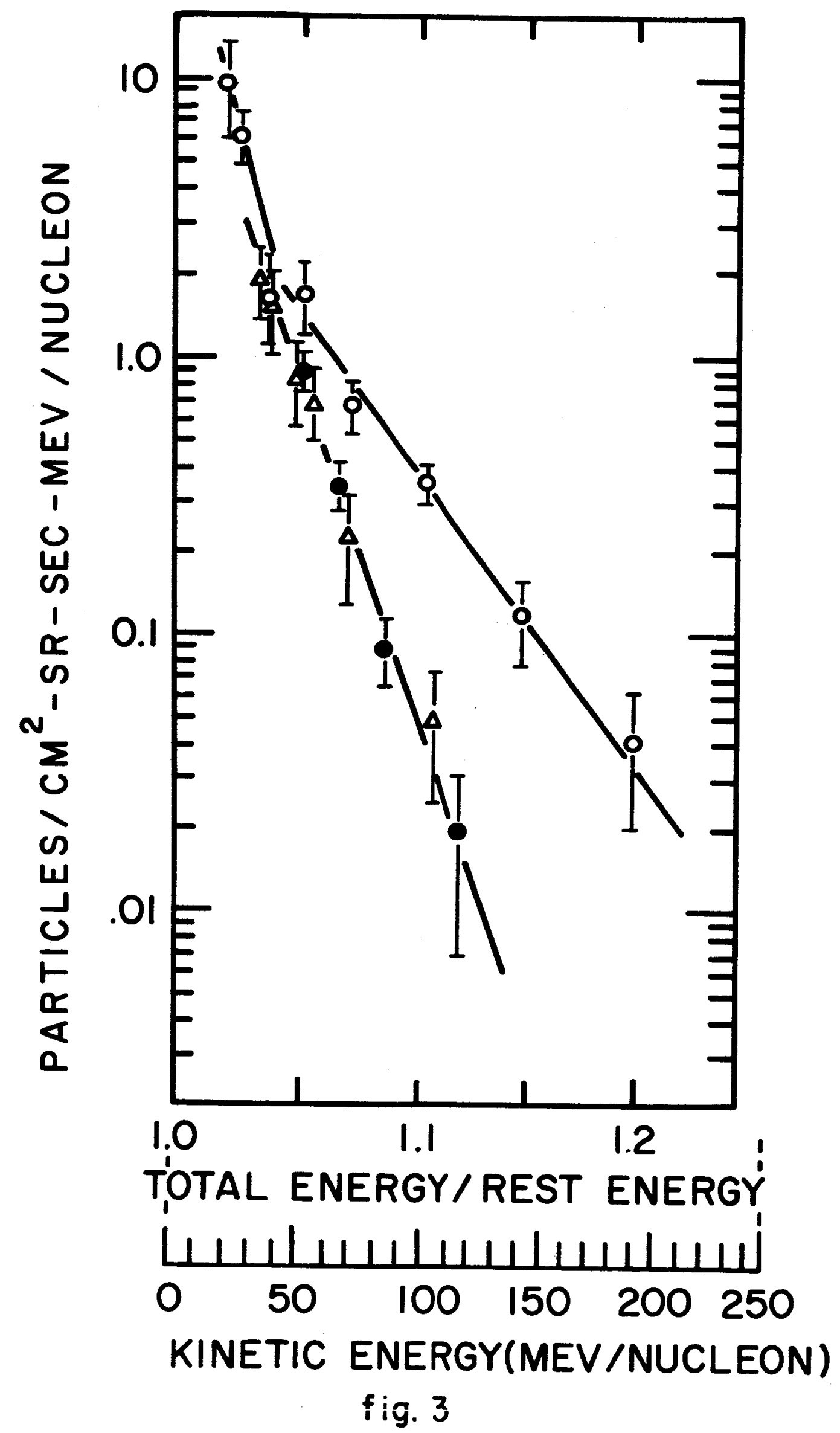




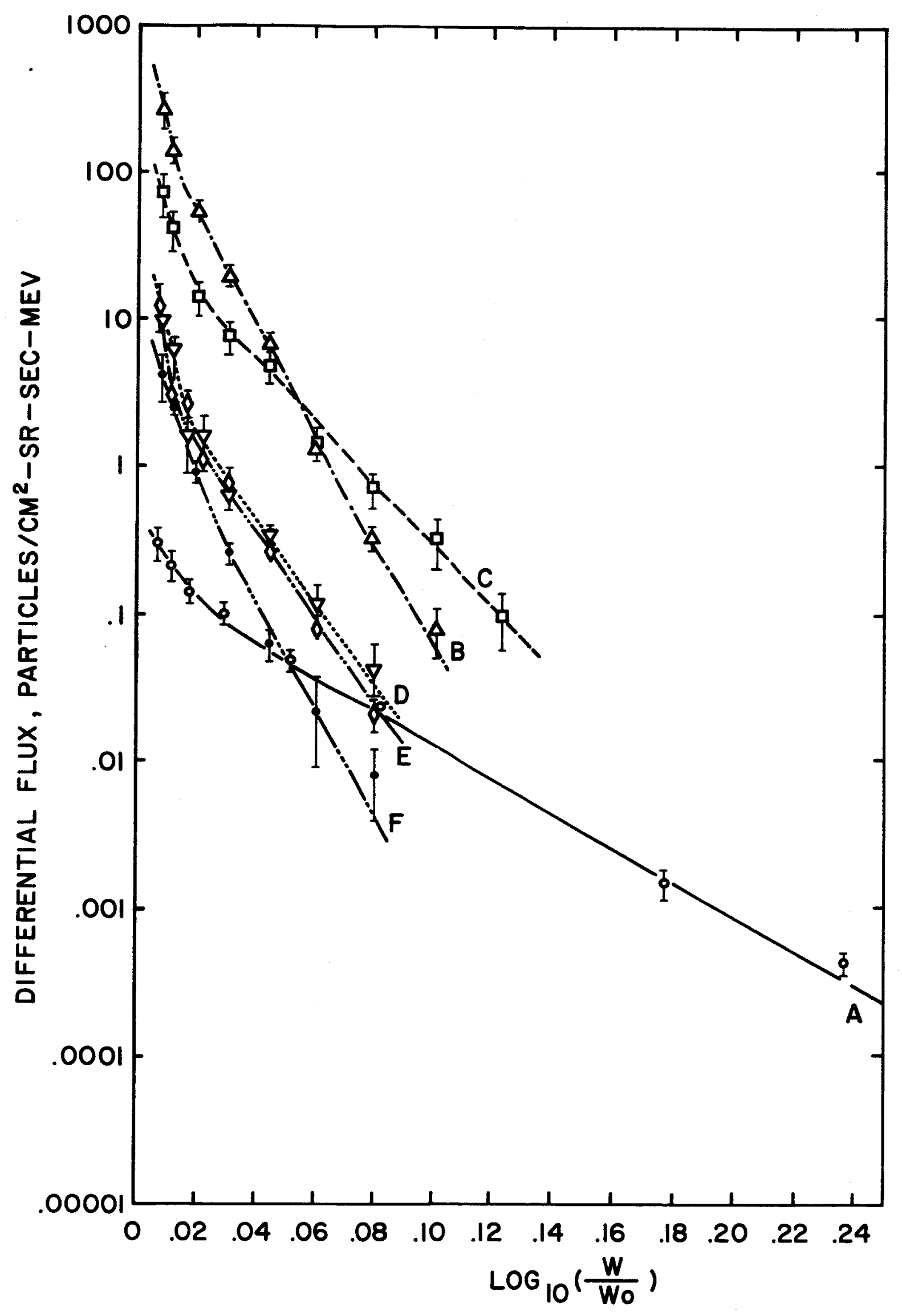

fig. $4 a$ 


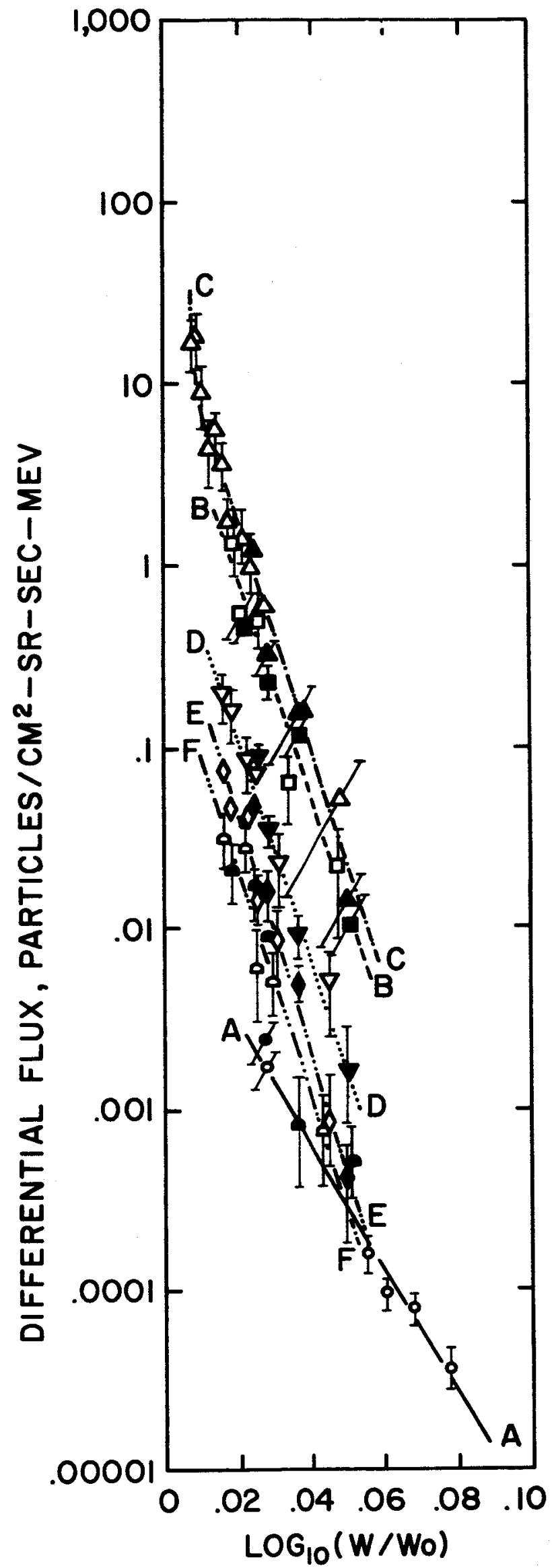

fig. 4 b 


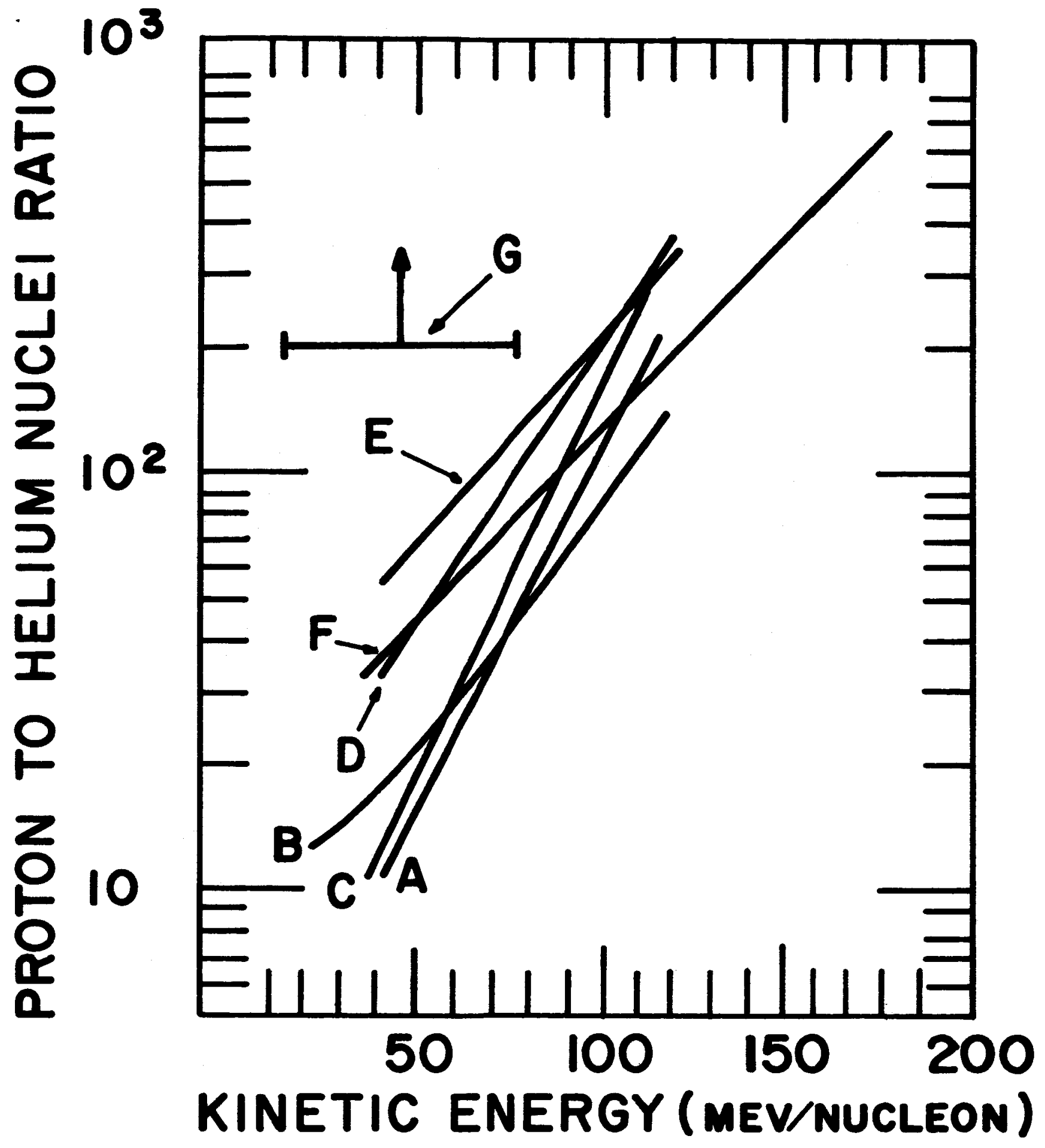

fig. 5 
Exrace and Changes for

compostriou of somar cositc ents

by

s. BLewas and C. B. Plchtel

N65 31552

Page 2, Ilne 1

$\therefore$

Change "1957 to 1962)." to "the yeare 1957 through 1962)."

Page 3, line 3

Change "Yagoda, 1962" to "Yagoda, F11x, and Pukul, 2961"

Page 5, Iine 1

Change "experimente" to "experimental systeme"

Page 5, Ilne 20

Change "proton dLffera" to "proton $\left(B^{2}\right)$ differe"

Page 6, equations (1) and (2)

Beplace " $X "$ in " $Y(\Lambda, X, T) "$ and " $R_{0}(\Lambda, X, X) "$ by "Z"

Page 6; second to last line

Change "1405" to" $1408 "$

Page 7, line 3

Change "--chance of $R_{0}$ velues of protong-n" to "-mchance for the

Ro values of the protongen

Page 7, line 10

Chenge "-15 tp 300-a" to "t-15 to 300-m

Page 7 L Line 16

Change "0.8 Mev" to "0.8 Bv"

Page 7, line 21

Change "Biswas and Fichtel (1963)" to "Biswas and Fichtel (1964)" 
Page 9, line 6

Change "-Flchtel (2963) v1th-d to "-Flchtel (2964) and vitb-a"

Page 10, line 18

Change "charge" to "charged"

Page 10, line 22

Change "medlum muclel (6 $\leq$ nucleax charge $\leq 9)-4$ " to "madlum nucle1, $M,(6 \leq 2 \leq 9)^{\prime \prime}$

Pase 11, 11nes 8-11

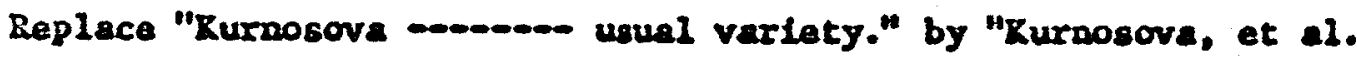

(1962) have reportad a few two to twenty mlnute increases of heavy nuclel above cosmic ray background in the ralatfolotic reglon, not assoclated with a solar particle event of the ieual varlety."

Pege 11, Ine 13

Change "experimentg" to "experimental systems"

Zage 11, last line

Delete "subsequent Iy"

Page 12, line 3

Change "proton to deuteron" to "deuteron to proton"

Page 12, Ine 7

Change "-- sam energy" to "camb energy/"

Page 13, Ine 4

Change " $4 \times 10^{9}$ (estlmated by Lal, Rajagopalan, and Venkatavaradan," to " $4 \times 10^{9} / \mathrm{cm}^{2}$ (estimated by Lel, Rajagopalan, and Venkatovaradan," 
Page 14, Iine 14

Change "0.2 with" to "0.2 at about $50 \mathrm{Her} /$ mucleon w1th"

Page 15, line 5

Change "lose thaix enargy" to "lose most of thelr kinetic energ"

Page 15, line 15

Change "posltive of electrone" to "posttive evidence for electrons"

Page 15, Line 16

Change "0.0025 for the rigldity" to "0.0025 for the electron to

proton ratio in the rigidity"

Fage 15, gecond to last line

Change "cont lnum" to continumen"

Page 16, second to lest 21 ne

Change "noutron frod" to"neutrons arising from"

Page 16, Ilnes 12

Change "> 1 Mev" to "< 1 Mev"

Page 16, last line

Change "suggested In the Ilterature" to "suggested (SImpson, 1963)."

Page 17 . Ines 17 and 18

Change "continuous quiet the flux" to "continuous quiet tim flux of low energy protons which lnereased at 10 energles"

Page 17 , second to last I1ne

Change "Plchtel, Guss, and Durgaprasad" to"Durgaprasad, PLchtel, and Guss"

Page 18, llne 21

Change "positron" to "posit10n" 
Page 20, line 6

Change "propogation" to "propagat 1on"

Page 20, 1lines 9 and 10

Change "-flux lo often, or usually, seen-o" to" "-plux lo usully seen-an

Fage 21, last line

Chenge "of $\mathrm{B}^{\mathrm{N}}$ to "of hydrogen"

Psge 22, Ine 8

Change "-. $\sigma\left[\mathrm{HQ}^{4}(\mathrm{p}, \mathrm{d}) \mathrm{a}^{3}\right]$ uas assumed to be $30 \mathrm{mb} .4$ to

$"--\sigma\left[\mathrm{He}^{4}(p, 2 p) \mathrm{B}^{3}\right]$ was assumed to be $10 \mathrm{mb}$ ".

Page 22, llae 10

Change "deutron" to "deuterons"

Page 22, second to last Ifne

Change "6-42. which Ld" to "6-4 $6_{-4}^{+12} \mathrm{gm} / \mathrm{cm}^{2}$, which--"

Page 23, line 2

Change "He 3 " to "Re 3 nuclet"

Page 23,1 ine 3

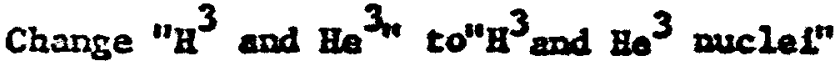

Page 23, line 17

Change "amoung" to "anount"

Page 23, I1ne 19

Change "by detectors 10 " to "from detectors flown la"

paze 24, line 21

Change "at tbe sun" to "of the solar atmosphere"

Paze 25, line 11

Change "--flelds (Sweet-" to"--flelds at a neutral point (Glovanelli, 1948; Sweet-m" 
Pane 25, IIne 14

Change "thermonuleax" to "tharmonuclear"

Page 26, Ilne 22

Change $m \mathrm{M} / \mathrm{Z}^{\prime \prime}$ to "the mase to charge rat1o"

Page 26, Llng 24

Delete. "for exenole"

Pase 27 , last line

Chenge "Ho" guggests there" to "Bo" nuclel suggests that there"

Page 29, Line 13

Change "Parker. 1957" to "Perker $1957 \mathrm{a}$ "

Page 29. line 18 to 20

Replace "However, elnce the early-mo-trua value." by "Other experimental evidence, lncluding the very proupt arrival of high enargy solar particles in many events. suggests that the major portion of the acceleration is completed within a few minutes."

Page 29 , second line from bottom

Change "--Before processing further," to "--Before proceedlag Earther,"

Page 29 , second to last line

Change "sun" to"solar atmosphere"

Page 30,1 ines 4 and 7

Change "larger" to "heavlex"

Page 30, line 19

Change "about twenty" to "about charge twenty" 
Pese 33, Iine 8

Cinange " $1: 10^{3, \text { to }}$ " $10^{3}: 1 "$

Eage 33, line 22

Change "stars" belng" to "starst remains as a serlous objection to ordinary stars belog

Pare 33, Line 24

Change "Syroratsky" to "Syrovatsky"

Pane 34. 1ine 9

Change "sun" to"solar atwosphere"

Eare 34,1 lne 15

Change "deutrons" to "deuterons"

Ease 35, line 17

Chenge "deutron, tritons, Ho", electrons" to "deuterons, tritons, $\mathrm{Be}^{3}$, muclei, electrons"

References, page 1

After "Aller, I. H.: 1953-m" add "Alier, L. H.: 1961, The Abundance of the Elements, Intersclence Publishers, New York.

Refercaces, page 2

Cisange "Zlchtel, G. R., D. B. Guse, and I. Durgaprasad, 1965: to be published" to "Durgaprasad, N., C. E. Flchtel, and D. E. Guss: 1965, to be published."

IEile 2, heading on colums 5, 6, 8, 9 and footrotes * and t*

Change " $R(1, j, k)$ " to "RA(1,j,k)"

Table 3. heading on colum 3 and footnote *

Change "R(1,j,k)" to "RA(1,j,k)" 
Table 3. second footnote (t+t)

Change "factor to two" to "factor of two"

Table 5, first footnote $(t)$

Change "0.5 sec." to "0.5."

Change "Coldberg, Muller and Aller, 1962" to "Goldberg, Nuller,

and Alle $x, 1960^{\prime \prime}$

Captions for Elgures, Fig. I, line 3

\&1g, 2, line 3

Change" (Mediun Nuclei) 60" to"(Mediun Nuclei) × 60"

Captions for Elgures, Fig. 3 , line 5

Change "He Nuclei 10;0: (Medium Nucle1) 600" to" (He Nuclel) $\times 10$;0: (Medium Nucles) $\times 600^{\prime \prime}$

Captions for Figures, F1g. 5,1 ine 4

Chance "--(1963), Blowas, (1961), and Biswas and Fichtel (1963)-_" to "--(1963), and Biswas and Fichtel (1964)--" 


\section{Addit lonal Errate and Changes for}

COMPOSITION OF SOLAR COSMC RAYS

by

S. Blswas and C. B. Flchtel

\section{Page 3, line 4}

Change "--Blows and Fichtel, 1962--" to "--Biswas and Flchtel, $1964--3$

Page 7, line 2

Chenge "Biswas and Fichtel, 1963" to "Blswas and Fichtel, 1964" Page 15, line 17

Change "Mev/c" to "Yw"

References, page 1

Change "Blswas, S. and C. E. Plichtel: 1963" to "B1swas, S. and

C. E. Fichtel: 1964"

Isble 1, heading to third major colum

Change "Ro" to "Ro (Ifv)"

Table 1, second through fourth minor columns

Delete minus $\$$ ign before all numbers

Table 2, heeding to colum ?

Change "BeV/c" to "Bv" 